Revista Brasileira de Cartografia

ISSN 1808-0936 | https://doi.org/10.14393/revbrascartogr

Sociedade Brasileira de Cartografia, Geodésia, Fotogrametria e Sensoriamento Remoto

\title{
Sensibilidade Espectral dos Índices de Vegetação: GNDVI, NDVI e EVI na Mata Ciliar do Reservatório de Serrinha II - PE, Brasil
}

\author{
Spectral Sensitivity of Vegetation Indices: GNDVI, NDVI and EVI in the Riparian Forest \\ of the Serrinha II Reservoir-PE, Brazil
}

Ubiratan Joaquim da Silva Junior ${ }^{1}$, Rodrigo Mikosz Gonçalves ${ }^{2}$, Leidjane Maria Maciel de Oliveira ${ }^{3}$ e Juarez Antonio da Silva Junior 4

1 Universidade Federal de Pernambuco, Programa de Pós-graduação em Ciências Geodésicas e Tecnologias da Geoinformação, Departamento de Engenharia Cartográfica, Recife, Brasil. ubiratan.joaquim@ufpe.br.

ORCID: https://orcid.org/ 0000-0001-7995-6416

2 Universidade Federal de Pernambuco, Programa de Pós-graduação em Ciências Geodésicas e Tecnologias da Geoinformação,

Departamento de Engenharia Cartográfica, Recife, Brasil. rodrigo.mikosz@ufpe.br.

ORCID: https://orcid.org/ 0000-0002-5066-1910

3 Universidade Federal de Pernambuco, Programa Pós-graduação em Ciências Geodésicas e Tecnologias da Geoinformação,

Departamento de Engenharia Civil e Ambiental, Recife, Brasil. leidjane.oliveira@ufpe.br.

ORCID: https://orcid.org/ 0000-0003-1251-6998/

4 Universidade Federal de Pernambuco, Departamento de Engenharia Cartográfica, Recife, Brasil. juarez.silvajunior@ufpe.br. ORCID: https://orcid.org/0000-0002-2898-0309

Resumo: Matas ciliares são florestas associadas a córregos, rios e lagos possuindo influência na proteção dos ecossistemas de ações antrópicas. O objetivo deste estudo foi identificar as alterações nas áreas de mata ciliar da Caatinga, a partir da sensibilidade espectral dos índices de vegetação (IVs): GNDVI, NDVI e EVI, considerando uma análise espaço temporal (1985 a 2019) no entorno do Reservatório de Serrinha II - PE - Brasil. Para isso, foram utilizadas imagens dos satélites TM - Landsat 5, OLI - Landsat 8 e MSI - Sentinel 2. Os IVs foram validados comparando perfis espectrais e correlação dos produtos gerados pelo Landsat 8 e Sentinel 2. Através da classificação binária dos índices, foram identificadas as alterações na cobertura vegetal, entre 1996 e 2019, resultando uma perda de 55,40\% de vegetação. Estes eventos, associados aos efeitos da seca também foram observados para o reservatório, entre 2012 e 2019, com 32,36\% de redução do espelho d'água. Entre os IVs, o GNDVI e NDVI apresentaram maior sensibilidade para identificar a mata ciliar com coeficiente de Pearson igual a 0,93 para o Landsat 8; 0,94 para o Sentinel 2 e acurácia global superior a 95\%. Por outro lado, o EVI mostrou-se mais sensível para identificar a área do reservatório, com acurácia global de 99,48\%. O monitoramento de reservatórios artificiais e mata ciliares de florestas sazonalmente secas como a Caatinga se beneficiam do uso de IVs onde a escolha de um determinado IV isolado deve ser interpretada com cautela de acordo com as características da região estudada.

Palavras-chave: Índices de Vegetação. Classificação Binária. Matas Ciliares. Caatinga. Reservatórios.

\begin{abstract}
Riparian forests are forests associated with streams, rivers and lakes that have an influence on the protection of ecosystems from man-made actions. The objective of this study was to identify changes in the Caatinga riparian forest areas, based on the spectral sensitivity of vegetation indices (IVs): GNDVI, NDVI and EVI, considering a temporal space analysis (1985 to 2019) in the surroundings of the Serrinha II Reservoir - PE - Brazil. For this purpose, satellite images from TM - Landsat 5, OLI - Landsat 8 and MSI - Sentinel 2 were used. The IVs were validated comparing spectral profiles and correlation of the products generated by Landsat 8 and Sentinel 2. Through the binary classification of the indices, changes in vegetation cover were identified between 1996 and 2019, resulting in a loss of $55.40 \%$ of vegetation. These events, associated with drought effects were also observed for the reservoir, between 2012 and 2019, with $32.36 \%$ reduction in the water mirror. Among the IVs, the GNDVI and NDVI showed greater sensitivity to identify the riparian forest with a Pearson coefficient equal to 0.93 for Landsat 8; 0.94 for Sentinel 2 and an overall accuracy of over $95 \%$. On the other hand, EVI was more sensitive to identify the reservoir area, with overall accuracy of $99.48 \%$. The monitoring of artificial reservoirs and riparian forests of seasonally dry forests such as the Caatinga benefit from the use of IVs where the choice of a given isolated IV should be interpreted with caution according to the characteristics of the region studied.
\end{abstract}

Keywords: Vegetation Indexes. Binary classification. Riparian forests. Caatinga. Reservoir. 


\section{INTRODUÇÃO}

As matas ciliares são consideradas de imensa importância para a continuidade de biomas como a Caatinga no nordeste do Brasil. Essas áreas têm sido modificadas nas últimas décadas por ações antrópicas, que vem alterado significativamente o cenário natural (VALERA et al., 2019), sendo ameaçadas de extinção no Brasil, devido ao desmatamento generalizado para agricultura e extração mineral (BARBOSA et al., 2019; CUNHA et al., 2019). Por este motivo é necessário o investimento em planejamento e programas que possibilitem análises do uso do solo e qualidade da água em áreas pertencentes à mata ciliares e sua interação com o meio antrópico (HÉNAULT-ETHIER et al., 2017).

Os recursos naturais da Terra e suas características se beneficiam do Sensoriamento Remoto, a fim de obter informações sobre sua detecção espacial, descrição, quantificação e monitoramento (KIMM et al., 2020; AREKHI et al., 2019). Os sistemas de Sensoriamento Remoto fornecem dados periódicos e consistentes da superfície terrestre sendo um instrumento fundamental no monitoramento global e uma das principais ferramentas usadas para entender os processos dinâmicos inerentes às diversas formas ecossistêmicas e suas respostas as mudanças climáticas (MERCIER et al., 2020; GHOLIZADEH et al., 2018; TESTA et al., 2018). Dentre as técnicas de processamentos de imagens obtidas por Sensoriamento Remoto têm-se os índices de vegetação (IVs), objetivando a exploração das propriedades espectrais da vegetação, a partir do comportamento da sua reflectância nas regiões do visível e infravermelho próximo, com aplicações para o monitoramento das matas ciliares e planejamento desses espaços (ZHANG et al., 2019; YANG et al., 2018; BECK, 2006). Entre eles destacam-se o GNDVI (Green Normalized Difference Vegetation Index), útil para avaliar as variações de biomassa verde na escala do dossel, o NDVI (Normalized Difference Vegetation) que é um indicativo da vegetação fotossinteticamente ativa (ZHOU; ZHONG, 2020; ALI et al., 2019) e o EVI (Enhanced Vegetation Index), utilizado como estimador de parâmetros biofísicos como a evapotranspiração (JARCHOW et al., 2018).

Zhou e Zhong (2020) utilizaram os IVs: GNDVI, EVI e NDVI para qualificar o comportamento de crescimento da vegetação, através de um modelo de mistura de refletância da superfície, com base no algoritmo Kalman Filter Reflectance Fusion Model (KFRFM), para prever incertezas em imagens sintéticas da série temporal dos satélites Landsat e MODIS (Moderate Resolution Imaging Spectroradiometer), em Ottawa, Canadá. Na região Nordeste do Brasil, Barbosa et al. (2019) analisaram os padrões espaço-temporais do impacto da seca na vegetação do bioma Caatinga, entre 2008 e 2016, empregando o NDVI, explorando a correlação entre o índice e a precipitação em escala local a regional, com séries temporais do Meteosat Second Generation (MSG).

A característica semiárida da Caatinga oferece uma oportunidade única para estudar a vegetação em resposta às secas recorrentes, devido aos seus impactos negativos na erosão do solo (BARBOSA et al., 2019). No entanto, a resposta da vegetação da Caatinga, aos IVs existentes, ainda é solicitante de modelagens, validações e estudos. Apesar de existirem 144 IVs, sendo 21 de banda larga e 123 de banda estreita (TONG; HE, 2017), eles não foram criados para o bioma Caatinga, que possui um grau notável de heterogeneidade vegetal em decorrência da dinâmica do déficit pluviométrico existente na região. Isto se torna ainda mais escasso, em estudos que correlacionem modelagens dos IVs já existentes, e a vegetação da Caatinga para áreas de mata ciliar. Pois, além de sofrer com escassez pluviométrica natural da região, essas áreas possuem diferenças em termos de umidade e tipo do solo. Tornando-as de grande importância para a distribuição hídrica, manutenção do ciclo hidrológico das bacias do semiárido, qualidade da água e sobrevivência dos ecossistemas que integram a relação: vegetação e corpo hídrico.

Portanto, os objetivos deste estudo foram: (i) Investigar possíveis alterações no cenário da cobertura vegetal da mata ciliar do bioma Caatinga, no entorno do Reservatório Serrinha II em Pernambuco, utilizando os índices de vegetação (IVs): GNDVI, NDVI e EVI, numa série temporal dos satélites TM - Landsat 5 e OLI - Landsat 8, entre 1985 e 2019, (ii) Analisar a sensibilidade dos IVs que mais se aproximem às características fenológicas desta região e (iii) Comparar os resultados obtidos pelos IVs através das análises de perfis espectrais e correlação com entre os produtos gerados pelos satélites Landsat 8 e MSI - Sentinel 2. 


\section{MATERIAIS E MÉTODOS}

\section{1 Área de Estudo}

No Brasil, as matas ciliares são denominadas Áreas de Preservação Permanente (APP), nos termos dos $\operatorname{artigos} 4^{\circ}, 5^{\circ}$ e $6^{\circ}$ da Lei no $12.651 / 12$ (Código Florestal) (BRASIL, 1965), sendo definidas como: área coberta ou não por vegetação nativa, com a função ambiental de preservar os recursos hídricos, a paisagem, a estabilidade geológica e a biodiversidade, facilitando o fluxo gênico da fauna e flora, protegendo solo e garantindo o bem-estar das populações humanas.

O experimento deste artigo foi realizado tendo como área de estudo a mata ciliar do Reservatório de Serrinha II, construída em 1996 pelo Departamento Nacional de Obras Contra as Secas (DNOCS), localizado no Semiárido Pernambucano, no município de Serra Talhada (SILVA et al., 2017), de coordenadas geográficas ( $\left.08^{\circ} 11^{\prime} 58,64^{\prime \prime S}, 38^{\circ} 32^{\prime} 31,98^{\prime \prime W}\right)$. Este é o segundo maior reservatório do estado, com capacidade máxima de 311,08 $\left(\mathrm{hm}^{3}\right)$ de água, pertencente à Bacia Hidrográfica do rio Pajeú.

A vegetação predominante é a Caatinga, única no Brasil, sendo a maior floresta tropical sazonal seca contínua com vegetação florestal nas Américas (CUNHA et al. 2019), caracterizada por um mosaico de vegetação xerofítica que se estende por cerca de $735.000 \mathrm{~km}^{2}$ através do Nordeste Brasileiro (LEAL et al., 2003) com árvores e arbustos baixos, ramificados, espinhosos, que emergem da folhagem microfílica e decídua na estação seca (MARIANO et al., 2018). Barbosa et al. (2019) afirmaram que a vulnerabilidade da vegetação da Caatinga à secas periódicas é exacerbada por altos níveis de degradação do habitat.

A Figura 1 A apresenta a extensão do Bioma Caatinga sobre o Brasil. A Figura 1 B, apresenta um esboço da localização geográfica da área de estudo, inserida no município pernambucano de Serra Talhada, Região Nordeste do Brasil. E a Figura 1C uma composição colorida RGB da área de estudo, detalhando o corpo hídrico e Mata Ciliar e a localização de 2 perfis espectrais utilizados posteriormente no item 3.3.1.

Figura 1 - Área de Estudo. (A) Área de Estudo inserida no Bioma Caatinga, Brasil. (B) Localização do Reservatório no Estado de Pernambuco, Nordeste Brasileiro. (C) Cena do Satélite MSI - Sentinel 2 de 17 de Novembro de 2019.
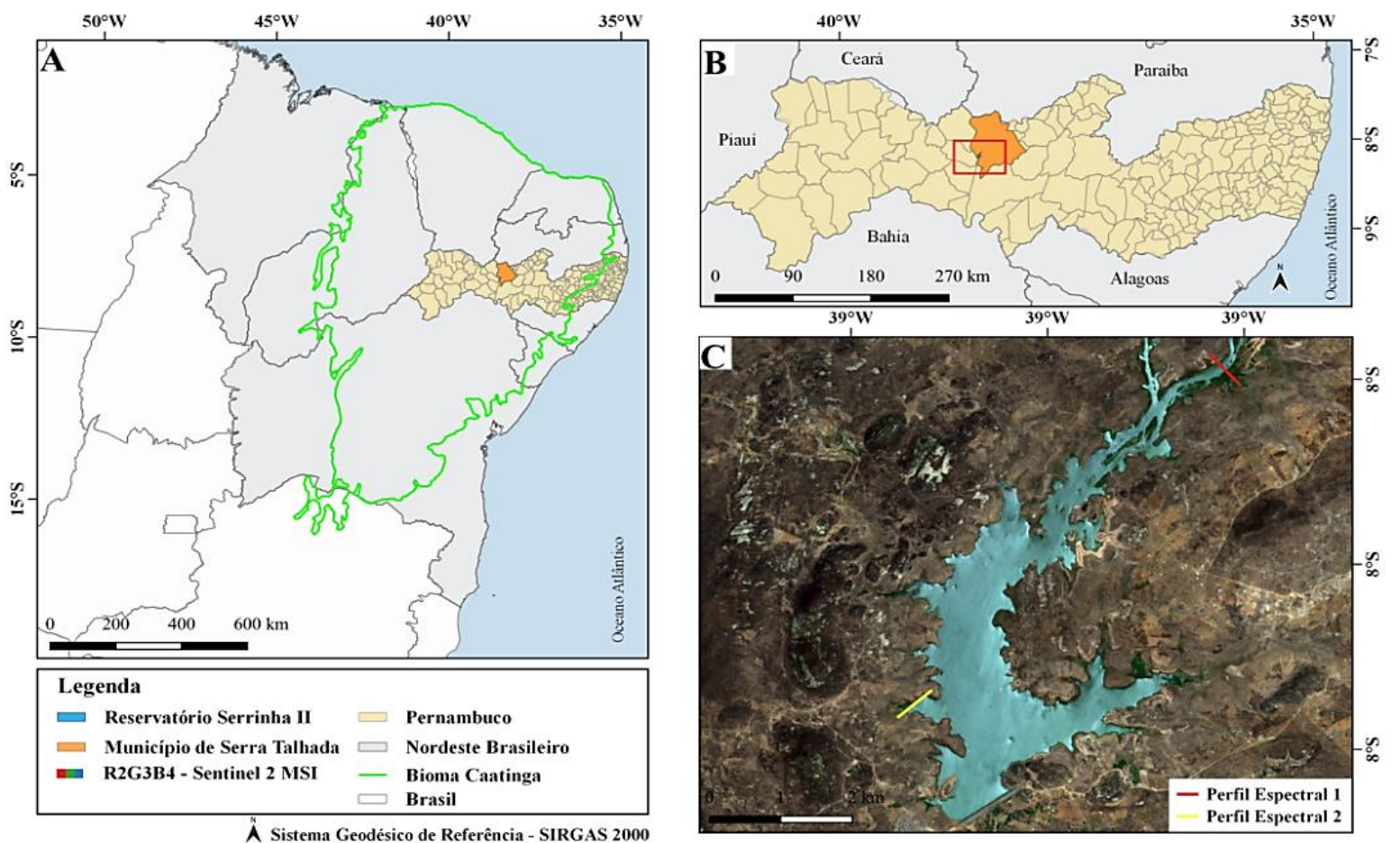

Fonte: Os autores (2021).

\subsection{Materiais}

a) Imagens Orbitais

Foram utilizadas cenas de imagens orbitais dos satélites TM - Landsat 5, OLI - Landsat 8 e MSI - 
Sentinel 2, conforme as características apresentadas na Tabela 1. As imagens foram obtidas para o período de baixa incidência pluviométrica da região de estudo, que compreende os meses de junho a dezembro, escolhidos a fim de identificar possíveis atenuantes de seca na mata ciliar. Segundo Chaves et. al. (2008) e Bispo, Valeriano e Duplich (2010) o período seco tem se definido como a melhor época para se realizar o diagnóstico e o mapeamento da vegetação de Caatinga.

Tabela 1 - Características das imagens.

\begin{tabular}{|c|c|c|c|}
\hline \multirow[b]{2}{*}{ Características } & \multicolumn{3}{|c|}{ Sensor/Satélite } \\
\hline & $\begin{array}{l}\text { TM - Landsat } 5 \\
\text { (Thematic Mapper) }\end{array}$ & $\begin{array}{c}\text { OLI - Landsat } 8 \\
\text { (Operational Land Imager) }\end{array}$ & $\begin{array}{c}\text { MSI - Sentinel 2 } \\
\text { (Multispectral Instrument) }\end{array}$ \\
\hline Data de Aquisição & $18 / 11 / 1985$ e $15 / 10 / 1996$ & $16 / 11 / 2019$ & $17 / 11 / 2019$ \\
\hline Recobrimento Espectral & 0,450 a $12,50(\mu \mathrm{m})$ & 0,443 a $12,005(\mu \mathrm{m})$ & 0,443 a $2,190(\mu \mathrm{m})$ \\
\hline Resolução Radiométrica & 8 bits & 12 bits & 12 bits \\
\hline Resolução Espacial & $30(\mathrm{~m})$ & $30(\mathrm{~m})$ & 10 e $20(\mathrm{~m})$ \\
\hline Resolução Temporal & 16 dias & 16 dias & 5 dias \\
\hline Fonte & $\begin{array}{c}\text { United States Geological } \\
\text { Survey (USGS) }\end{array}$ & $\begin{array}{l}\text { United States Geological } \\
\text { Survey (USGS) }\end{array}$ & $\begin{array}{l}\text { European Space Agency } \\
\text { (ESA) }\end{array}$ \\
\hline
\end{tabular}

Fonte: USGS, ESA (2020).

As imagens do satélite MSI - Sentinel 2 são complementares a imagens do OLI - Landsat 8, e os dois instrumentos partilham semelhanças nas especificações das bandas (SHAO et al., 2019), como descrito na Tabela 1. Para o mapeamento das áreas de mata ciliar, a utilização dos sensores em conjunto pode contornar limitações como: presença de nuvens, sombras, resoluções temporais e espacial e outros efeitos atmosféricos (FU; WENG, 2016). Destaca-se que a informação temporal pode detectar comportamentos típicos de matas ciliares como na resposta a inundações e períodos de seca (SKAKUN et al., 2014), identificação fenológica da vegetação (MELAAS et al., 2013) e detecção de alterações espaciais (WHITE et al., 2017).

b) Dados hidrológicos

Os dados de precipitação foram adquiridos através de estações de monitoramento da Agência Nacional de Águas e Saneamento (ANA) e Agência Pernambucana de Águas e Clima (APAC), conforme Tabela 2:

Tabela 2 - Dados hidrológicos para região de estudo, anual e durante o período de aquisição das imagens orbitais.

\begin{tabular}{c|c|c|c|c|c}
\hline Ano & Precipitação anual (mm) & Mês & Precipitação mensal (mm) & $\begin{array}{c}\text { Dias de } \\
\text { chuva/mês }\end{array}$ & Fonte \\
\hline 1985 & 1265 & Novembro & 0 & 0 & ANA - estação N N $^{\circ} 838015$ \\
1996 & 896,1 & Outubro & 9,4 & 2 & ANA - estação No 838015 \\
2019 & 527,4 & Novembro & 32,3 & 2 & APAC - estação N 505 \\
\hline
\end{tabular}

Fonte: ANA, APAC (2020).

\subsection{Métodos}

A metodologia empregada foi realiza em três etapas: na primeira etapa foi realizada a aquisição de dados com a seleção das cenas, em que, consideraram-se fatores como: ampla cobertura, ausência de nuvens, períodos com baixa incidência pluviométrica e disponibilidade de compartilhamento dos dados em nuvem. A segunda etapa trata-se do processamento digital das imagens. Feito isso, foram elaborados os cálculos dos IVs que possuem como resultados as cartas imagem para cada ano investigado, contendo os índices e extração das áreas por meio do processo de binarização para as classes mata ciliar e água. A terceira etapa refere-se à validação dos dados, com a comparação entre os produtos gerados pelos satélites OLI - Landsat 8 e MSI Sentinel 2, através das análises de perfis espectrais e de correlação entre os IVs.

\subsubsection{PROCESSAMENTO DIGITAL DAS IMAGENS}

As imagens são processadas através das etapas: reprojeção cartográfica ao Sistema Geodésico de Referência - SIRGAS 2000, conversão de radiância para reflectância e a correção atmosférica através do método de subtração de objetos escuros DOS (Dark Object Subtraction; CHAVEZ, 1988), aplicada para os dados de reflectância no topo da atmosfera (TOA), através da seleção dos valores no histograma e posteriormente subtraídos de cada banda. 


\subsubsection{CÁLCULOS DOS INDÍCES BIOFÍSICOS E EXTRAÇÃO DAS ÁREAS}

Os IVs configuram-se como medidas radiometricas adimensionais que indicam abundância relativa e atividade da vegetação verde, índice de área foliar, porcentagem de cobertura verde, teor de clorofila, entre outros elementos (JESSEN, 2009) e são capazes de evidenciar variações sazonais, interanuais e de longo termo de parâmetros estruturais, fenológicos e biofísicos da vegetação (MERRICK et al., 2019). Os índices GNDVI e NDVI variam entre -1 e 1, conforme a Eq. (1) e Eq. (2):

$$
G N D V I=\frac{\rho_{\left(\lambda_{N I R)}\right.}-\rho_{\left(\lambda_{\text {GREEN })}\right.}}{\rho_{\left(\lambda_{N I R)}\right.}+\rho_{\left(\lambda_{\text {GREEN })}\right.}}
$$

onde, $\rho_{N I R}=$ infravermelho próximo e $\rho_{\left(\lambda_{\text {GREEN })}\right.}=$ banda do verde (GITELSON; KAUFMAN; MERZLYAK, 1997),

$$
N D V I=\frac{\rho_{\left(\lambda_{N I R)}\right.}-\rho_{\left(\lambda_{R E D)}\right.}}{\rho_{\left(\lambda_{N I R)}\right.}+\rho_{\left(\lambda_{R E D)}\right.}}
$$

onde, $\rho_{N I R}=$ Infravermelho próximo e $\rho_{\left(\lambda_{R E D}\right.}=$ banda do vermelho (ROUSE et al., 1973).

E o EVI, variando entre -1 e 1, conforme a Eq. (3):

$$
E V I=\frac{G\left(\rho_{\left(\lambda_{N I R)}\right.}-\rho_{\left(\lambda_{R E D)}\right)}\right)}{\left(L_{S}+C_{1} \rho_{\left(\lambda_{R E D)}\right.}+C_{2} \rho_{\left(\lambda_{B L U E)}\right.}+\rho_{\left(\lambda_{N I R)}\right)}\right)}
$$

onde, $\rho_{\left(\lambda_{N I R)}\right.}=$ infravermelho próximo e $\rho_{\left(\lambda_{R E D)}\right.}=$ banda do vermelho, $\rho_{\left(\lambda_{B L U E)}\right.}=$ banda do azul, $L_{S}=$ parâmetro de ajuste ao solo; $G=$ fator de ganho; $C 1$ e $C 2=$ coeficientes de ajuste para efeito de aerossóis na atmosfera. Os valores dos coeficientes utilizados na equação desenvolvida para obtenção do EVI foram: $L=$ $1, C 1=6, C 2=7,5$ e $G=2,5$ (HUETE, 1988; HUETE et al., 2006).

\subsubsection{ANÁLISES ESTATÍTICAS E VALIDAÇÃO DOS IVS}

Com a finalidade de estabelecer um processo de validação para os mapas gerados através dos IVs, utilizou-se como ferramenta: análise espectral dos IVs, correlação estatística e avaliação da reposta dos parâmetros acurácia global, índice Kappa, Acurácia do Usuário (Erros de omissão - EO) e Acurácia do Produtor (Erros de omissão - EO), para o satélite OLI - Landsat 8 tendo como referência o satélite MSI Sentinel 2, buscando investigar a aplicação de um processo de validação entre os sensores. Nesta etapa os resultados dos índices investigados foram comparados com o IV SAVI (Soil Adjusted Vegetation Index; HUETE, 1998), variando o parâmetro de ajuste ao solo ( $\left.\mathrm{Ls}_{\mathrm{S}}\right)$ de 0 a 1, conforme Eq. (4):

$$
S A V I=\frac{\left(1+L_{S}\right)\left(\rho_{\left(\lambda_{N I R)}\right.}-\rho_{\left(\lambda_{R E D)}\right)}\right)}{\left(L_{S}+\rho_{\left(\lambda_{N I R)}\right.}+\rho_{\left(\lambda_{R E D}\right)}\right)}
$$

onde, $\rho_{N I R}=$ Infravermelho próximo, $\rho_{\left(\lambda_{R E D}\right)}=$ banda do vermelho e $L_{S}=$ parâmetro de ajuste ao solo ( 0 a 1$)$.

Segundo Qi et al. (1994) é adotado valor 1 para áreas com baixa cobertura vegetal, 0,50 intermediárias, 0,25 para áreas com alta densidade, e ao assumir valor igual a zero, tem-se uma igualdade do índice com o NDVI.

O comportamento espectral dos IVs foi estudado a partir de perfis, traçados em áreas da mata ciliar ao longo do Reservatório Serrinha II, entre elas áreas de confluência entre o reservatório e o Rio Pajeú (Figura $1 \mathrm{C})$.

Para as análises estatísticas foram amostrados 100 pixels aleatoriamente, formando uma estatística zonal para cada índice através da média $(\sigma)$ e desvio padrão $(\alpha)$, distribuído nas áreas de mata ciliar para os 
Sensores OLI e MSI, identificados por meio de uma composição RGB da imagem. Utilizados para extração dos valores de reflectância aparente das bandas empregadas na álgebra de cada índice. Esta metodologia de amostragem e análise estatística é baseada em experimentos desenvolvidos por Zhou e Zhong (2020), Liu et al. (2018) e Loranty et al. (2018).

Em seguida, obteve-se a extração das classes: mata ciliar e água (reservatório), através do processo de binarização. Resultando nas áreas ocupadas pelas classes binarizadas para cada ano conforme o respectivo índice. O processo de binarização, ou seja, este tipo de classificação é baseada no modelo de presença e não presença. Dessa forma, o processo seguiu o critério de $(f(x, y)<\sigma)$, em que, o conjunto de pixels classificados recebe o valor 1 (presença) e $(f(x, y)>\sigma)$ recebe o valor de 0 (não presença). Onde, $f(x, y)$ é função que representa a imagem a ser binarizada gerada por cada índice, e $\sigma$ é a média obtida pela estatística zonal da seleção amostral realizada para as classes mata ciliar e água (reservatório). Dessa forma, além de separar as classes de interesse foi possível efetuar o cálculo de suas áreas.

Foi então, implementado um modelo de regressão linear, correlação Pearson $(\alpha)$ e coeficiente de determinação $\left(\mathrm{R}^{2}\right)$, utilizados como medidas de desempenho do modelo e comparação dos índices (HELMAN et al., 2018). Compararam-se os produtos das segmentações, através da avaliação da qualidade das classificações a partir do Índice Kappa e Exatidão Global, EC e EO.

\section{RESULTADOS E DISCUSSÃO}

\subsection{Variabilidade dos IVs}

Através da metodologia aplicada foram geradas cartas imagens dos sensores TM, OLI e MSI, representando a distribuição espacial dos índices: GNDVI (Figura 2A), NDVI (Figura 2B) e EVI (Figura 2C).

Figura 2 - Cartas imagem representando os IVs: A) GNDVI, B) NDVI e C) EVI para os Sensores OLI e MSI.

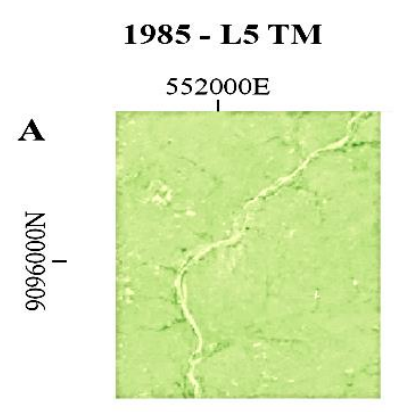

B

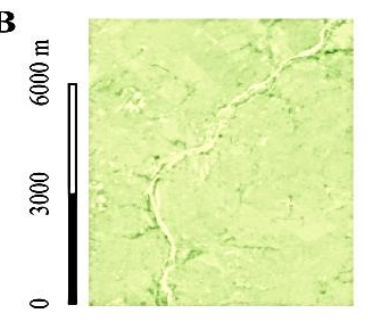

C

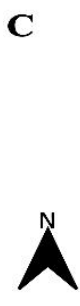

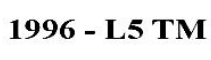
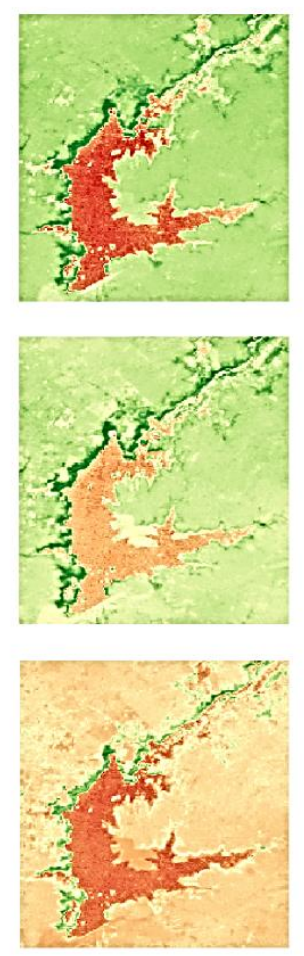

2019 - L8 OLI
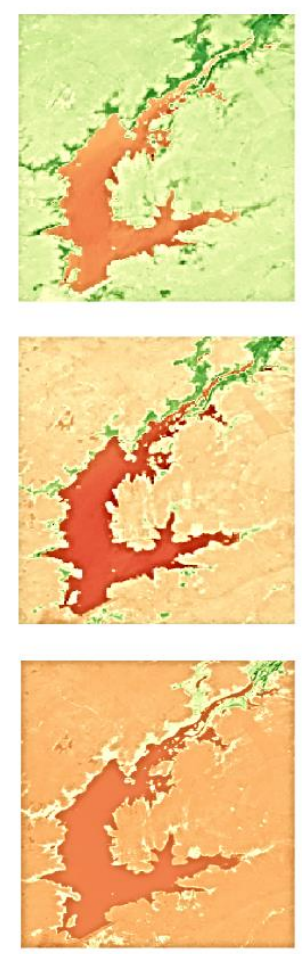

2019 - S 2 MSI
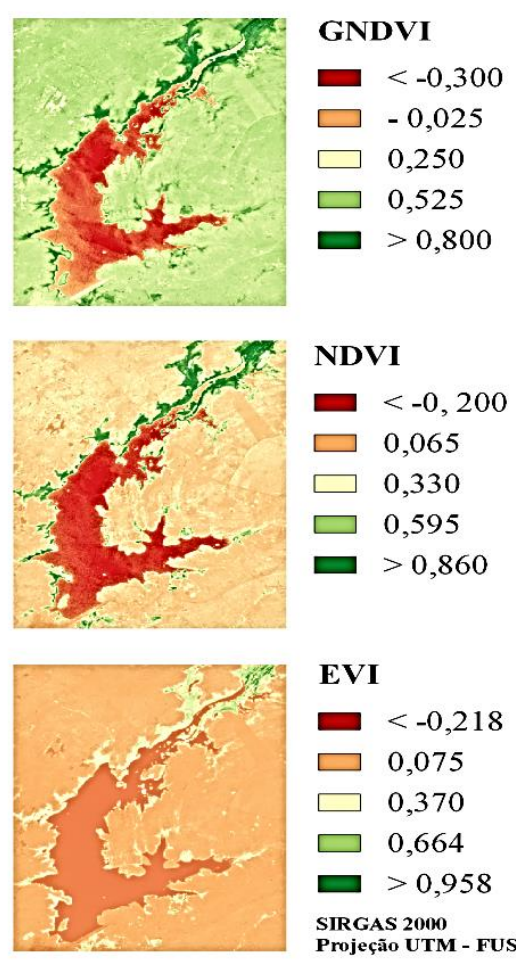

EVI

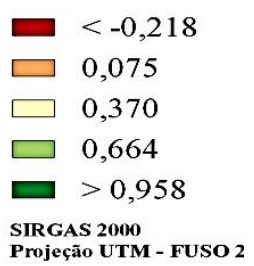

Fonte: Os autores (2021).

Ao analisar os resultados obtidos, as zonas com comportamento negativo são bem reconhecíveis (entre laranja e vermelho) e, as de comportamento positivo em que áreas onde a vegetação possui bons atributos de vigor para todos os índices (em verde).

O GNDVI (Figura 2A) apresentou uma amplitude de valores entre $-0,300$ a $>0,800$, em que grande 
parte da área analisada teve pixels com altos valores positivos de GNDVI, destes, os maiores valores concentraram-se nas áreas de mata ciliar. E as demais áreas, os pixels assumiram valores entre -0,020 e 0,250, representando possíveis áreas de vegetação rasteira. Estes resultados podem estar associados à redução do efeito de saturação nas áreas de vegetação densa ao substituir a faixa vermelha pela faixa verde (GNDVI em vez de NDVI) (NAVARRO et al., 2017). As áreas com maior cobertura vegetal, correspondente aos anos de 1985 e 1996, produtos do sensor TM, apresentaram maiores quantidades de pixels classificados com alto valor de GNDVI.

Para o NDVI houve uma redução da distribuição de pixels com auto vigor vegetativo nas áreas de mata ciliar entre os sensores, tornando-se mais evidente no ano de 2019, com valores máximo e mínimo, quando comparado ao GNDVI, variando entre $-0,200$ e > 0,860 obtendo altos valores para as áreas de mata ciliar, e baixos valores para corpos d'água nas demais regiões do recorte analisado, em sua maioria de extensão entre 0,065 e 0,330 .

Esses valores corroboram com os resultados encontrados em Cunha et al. (2019), em que o NDVI variou em média entre 0,13 a 0,73, e o EVI 0,08 a 0,57 para uma serie temporal de trinta anos, assim como, Morais et al. (2011), em áreas de transição de Caatinga, no município de Floresta (PE), encontrando valores de NDVI entre 0,208 a 0,803. Abade et al. (2015) obtiveram valores de NDVI de 0,80 $\pm 0,03$ para regiões com vegetação densa. Estudos realizado por Barbosa et al. (2019), mostraram que o índice de vegetação tem significativa correlação com as chuvas. Chaves et al. (2013), enfatizaram que valores de NDVI abaixo de 0,4 para o período seco são mais compatíveis para respostas da vegetação de Caatinga Hiperxerófila, estando de acordo como as condições empregadas a área de estudo do reservatório.

No mês de aquisição das imagens houve baixa incidência pluviométrica, conforme descreve a Tabela 2, o que pode ter corroborado com a resposta da redução de áreas com distribuição de pixels com altos valores de NDVI, porém, como regiões próximas a cursos hídricos tendem a ter elevada umidade, foi observado nestas proximidades um maior número de pixels com valores elevados de NDVI.

Dentre os índices, o EVI apresentou menores valores para a mata ciliar para o ano de 2019, com uma amplitude de $-0,218$ a > 0,958, por outro lado, os pixels com valores elevados foram encontrados em áreas de confluência do Reservatório. Nas demais áreas os pixels assumiram baixos valores, entre 0,07 e 0,37, ficando evidente o destaque entre as margens e o reservatório. Crespo-Mendes et al. (2019) afirmaram que o EVI é mais sensível às mudanças de cobertura do solo. Segundo, Macintyre, Niekerk e Mucina (2020) os índices podem sofrer variações devido a diversos fatores, e essas variedades dependem da interferência nos dosséis da vegetação em período de crescimento. Para Fu-min et al. (2007), em análises da vegetação por meio de imagens orbitais, o efeito da atmosfera deve ser levado em consideração. Embora a banda azul seja mais facilmente afetada pela atmosfera, a reflectância de alvos na superfície pode ser adquirida de forma precisa com o desenvolvimento de modelos específicos de correção atmosférica.

\subsection{Classificação binária da mata ciliar e reservatório}

Ao analisar a Figura 3, percebeu-se que no ano de 1985 com a não existência da barragem no reservatório, consequentemente da mata ciliar, a vegetação distribuiu-se de forma espaçada, porém com uma uniformidade nos pontos aproximando-se da região em que o Rio Pajeú tem curso. Em 1996 já foi perceptível à formação da mata ciliar em visão contínua, no entorno do reservatório. E em 2019, ainda presente, expressouse em baixa continuidade, possivelmente, uma redução espacial da mesma, ocasionada por efeitos de seca, baixa incidência pluviométrica ou ações antrópicas.

Em todos os anos analisados os IVs do infravermelho apresentaram maiores quantidades de pixels classificados para áreas de mata ciliar. Em 2019, o sensor MSI apresentou uma maior quantidade de pixels classificados pelo GNDVI e NDVI que o OLI. O EVI, apesar de em todos os anos não ter apresentado uma classificação relevante, havendo pouca representação da mata ciliar, em 1996 (Figura 3J), detectou uma maior porção de pixels classificados, o que possibilitou a formação de áreas da mata ciliar, diferindo do padrão seguido pelo índice para os demais anos. E em 2019, os resultados para o Landsat 8 e Sentinel 2, se mostraram com proximidade (Figuras 3I e L). 
Figura 3 - Extração das áreas de mata ciliar para os anos analisados.

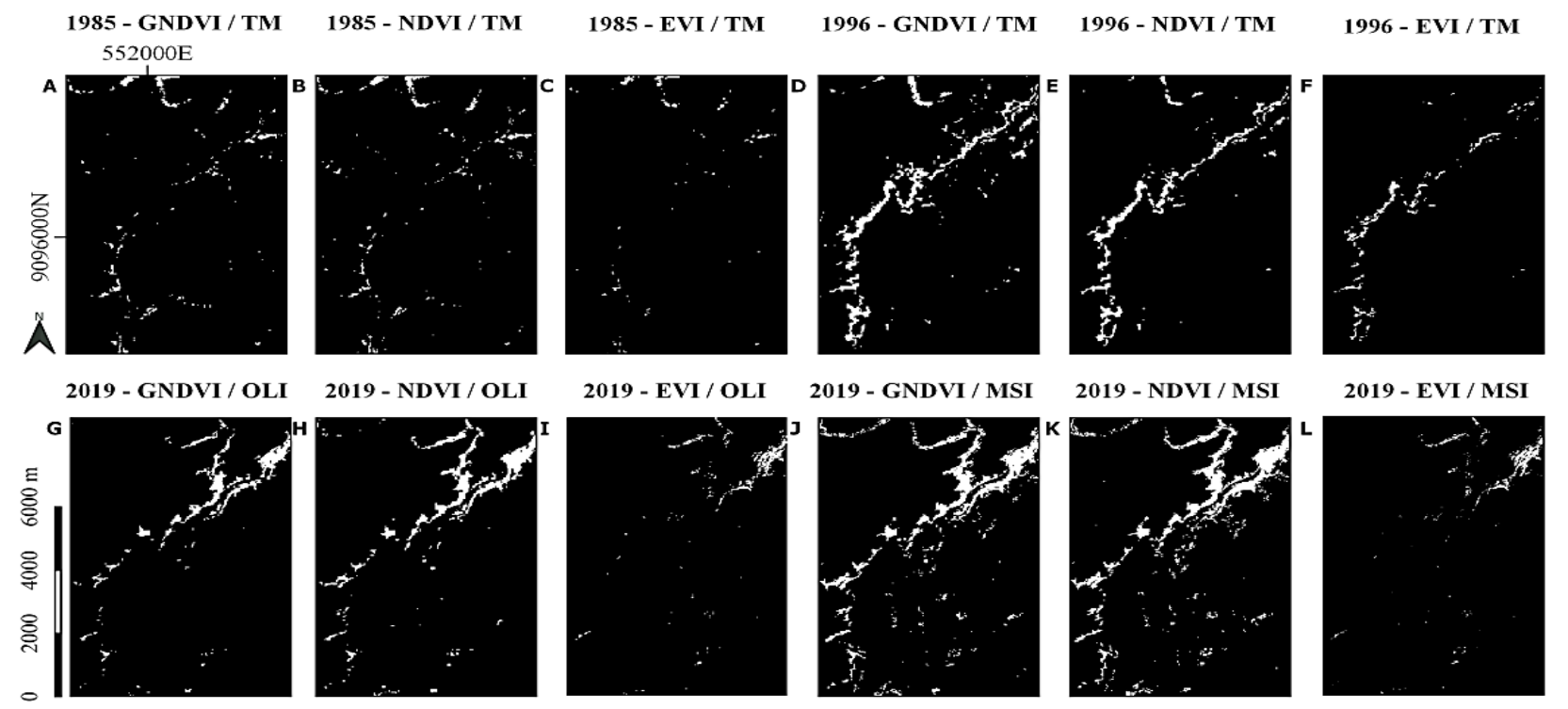

Fonte: Os autores (2021).

Para a detecção da área do reservatório os IVs apresentaram resultados considerados satisfatórios, como mostra a Figura 4.

Figura 4 - Extração das áreas do reservatório para os anos analisados.
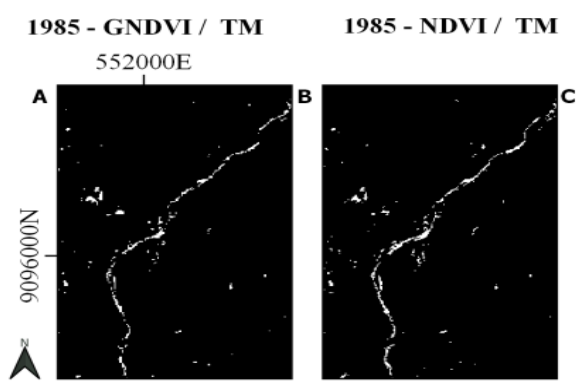

$1985-$ EVI / TM

1996 - GNDVI / TM

1996 - NDVI / TM

1996 - EVI / TM
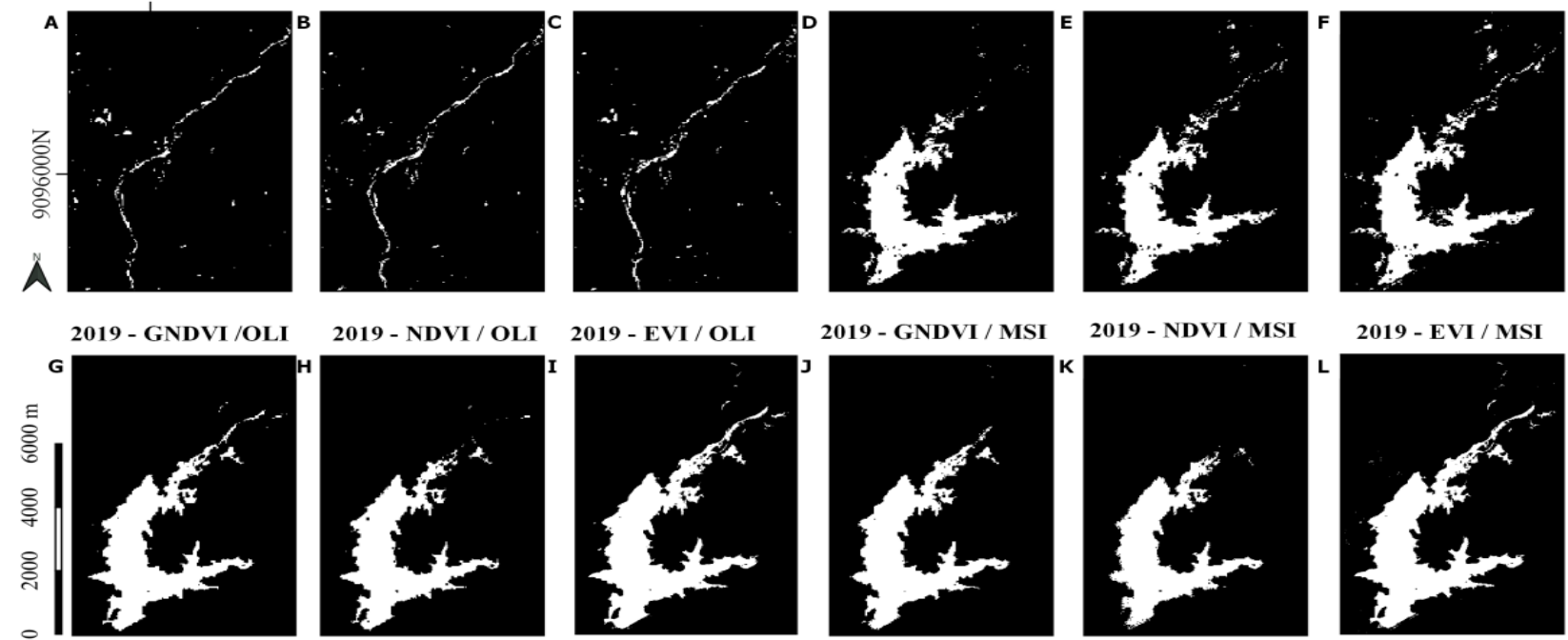

2019 - EVI / MSI

Fonte: Os autores (2021)

No ano de 1985 em todos os IVs foi possível identificar a presença do Rio Pajeú, porém foram classificados pixels além das áreas ocupadas pelo rio, sendo o NDVI (Figura 4A) e EVI (Figura 4B) os índices que apresentaram maiores quantidades, provavelmente, áreas de solo exposto ou com presença muito baixa de vegetação. Em 1996 o EVI (Figura 4F) e o NDVI (Figura 4E), tiveram a maior quantidade de pixels com a classe água, o que resultou no aumento de áreas do reservatório. Em 2019 o EVI (Figuras 4I e L) apresentou maior área, e o NDVI uma redução.

Esses resultados corroboram com a Tabela 3, que contem a porcentagem de área por índice ao longo dos anos analisados para as classes: mata ciliar e reservatório. Em que os IVs do infravermelho apresentaram maiores áreas para a classe mata ciliar, e o EVI para a classe reservatório. 
Tabela 3 - Áreas obtidas através do processo de binarização.

\begin{tabular}{|c|c|c|c|c|c|c|}
\hline \multirow{2}{*}{ Ano } & \multirow{2}{*}{ Sensor / Satélite } & \multirow{2}{*}{ Índice } & \multicolumn{2}{|c|}{ Mata Ciliar } & \multicolumn{2}{|c|}{ Reservatório } \\
\hline & & & Área (\%) & Área $\left(\mathbf{k m}^{2}\right)$ & Área (\%) & Área $\left(\mathrm{km}^{2}\right)$ \\
\hline \multirow{4}{*}{1985} & \multirow{3}{*}{ TM - Landsat 5} & GNDVI & 1,50 & 0,8289 & 1,34 & 0,7371 \\
\hline & & NDVI & 1,57 & 0,8658 & 1,53 & 0,8424 \\
\hline & & EVI & 0,65 & 0,3564 & 1,60 & 0,8793 \\
\hline & \multirow{3}{*}{$\mathrm{TM}$ - Landsat 5} & GNDVI & 7,80 & 4,3029 & 11,41 & 6,2667 \\
\hline \multirow[t]{2}{*}{1996} & & NDVI & 3,44 & 3,4021 & 11,56 & 6,3486 \\
\hline & & EVI & 1,31 & 1,3210 & 12,52 & 7,5623 \\
\hline \multirow{6}{*}{2019} & \multirow{3}{*}{ OLI - Landsat 8} & GNDVI & 4,34 & 2,3841 & 12,38 & 6,8013 \\
\hline & & NDVI & 3,06 & 1,6767 & 13,42 & 7,3701 \\
\hline & & EVI & 0,93 & 0,5139 & 14,74 & 8,0936 \\
\hline & \multirow{3}{*}{ MSI - Sentinel 2} & GNDVI & 6,18 & 3,3939 & 13,49 & 7,4079 \\
\hline & & NDVI & 6,56 & 3,6027 & 12,10 & 6,6456 \\
\hline & & EVI & 0,90 & 0,4959 & 15,00 & 8,2393 \\
\hline
\end{tabular}

Fonte: Os autores (2021).

O GNDVI foi superior aos IVs para a mata ciliar sendo superado pelo NDVI apenas nos anos de 1985 e 2019 para o satélite Sentinel 2. O NDVI por sua vez manteve-se com valores medianos para a mata ciliar, e EVI com valores baixos. Para a classe água o NDVI supera o GNDVI para os produtos derivados da série Landsat e, o EVI em todos os anos e satélites, apresentou maiores áreas para esta classe. Além disso, apesar dos anos de 1985 e 1996 apresentarem uma maior quantidade de pixels que não se enquadravam nas classes e foram classificados, devido aos limiares de cada índice, é perceptível uma redução visual de áreas de mata ciliar entre os anos de 1996 e 2019 em todos os índices.

Em síntese, pode se extrair que antes da construção do reservatório apesar da presença do Rio Pajeú na área existia uma maior cobertura vegetal que estava distribuída por além das proximidades do rio, e que ainda continua presente com o cenário pós-operação do reservatório. Os resultados para o ano de 2019 indicaram que apesar da presença de Serrinha II, não houve uma estabilidade de densidade florestal para além das áreas do entorno da barragem e sim uma redução indicando um possível evento de seca, mudanças climáticas ou ação antrópica ao longo dos anos. Além disso, fatores externos como operações inerentes a dinâmica do reservatório, à topografia do ambiente, podem proporcionar uma redução ou aumento da cobertura vegetal, e consequentemente influenciar nos dados.

\subsection{Validação dos IVs através da comparação entre os satélites Landsat 8 e Sentinel 2}

Em particular, o valor médio dos IVs reflete uma produtividade e biomassa médias, (CANDIAGO et al., 2015). O satélite MSI - Sentinel 2, inclui seis bandas de monitoramento terrestre comparável ao OLI Landsat 8 (Drusch et al., 2012). Segundo Meyer et al. (2019), devido a essas características e diferenças entre os sensores, é interessante entender qual sensor tem melhor desempenho na previsão da cobertura vegetal e da biomassa. Portanto, executou-se uma avaliação dos resultados gerados com IVs pelo satélite OLI - Landsat 8 com o satélite MSI - Sentinel 2 com imagens do mesmo ano, através dos perfis espectrais, correlação estatística entre os IVs e qualidade no processo de classificação das imagens por binarização para extração das áreas de mata ciliar e do reservatório.

\subsubsection{PERFIS ESPECTRAIS}

A Figura 5 apresenta o comportamento dos perfis espectrais, com localização destacada na Figura 1C, para os IVs comparando os sensores OLI e MSI. Tais perfis foram estabelecidos em pontos de concentração visual de vegetação densa nas áreas de mata ciliar.

Os IVs GNDVI (Figuras 5A e B) e NDVI (5C e D) em todos os perfis transversais apresentaram uma boa resposta da vegetação, para cobertura da mata ciliar, com valores próximos que variam de 0,6 a 0,8 , tendo o GNDVI assumido maiores valores que o NDVI. 
Figura 5 - Perfis Espectrais dos IVs para os Sensores MSI e OLI.

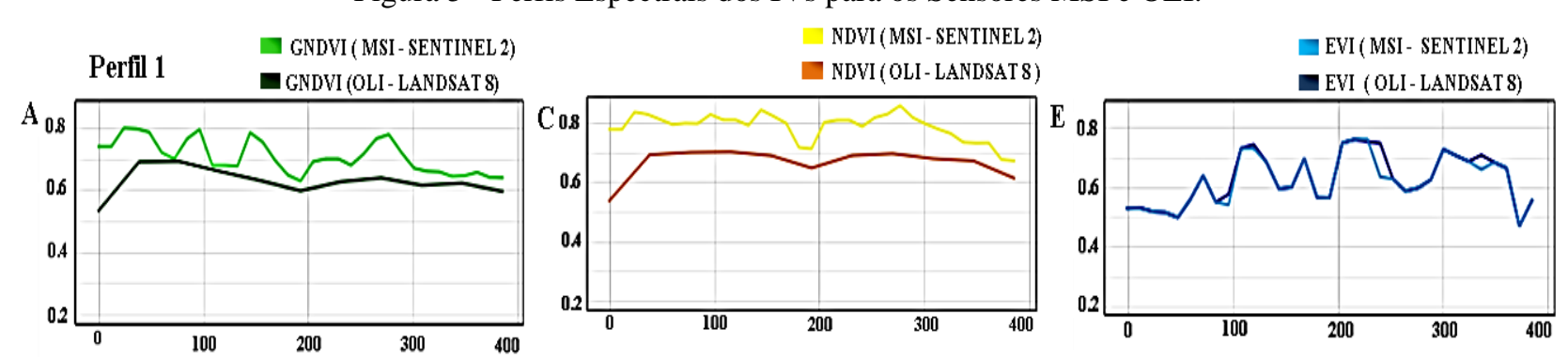

Perfil 2
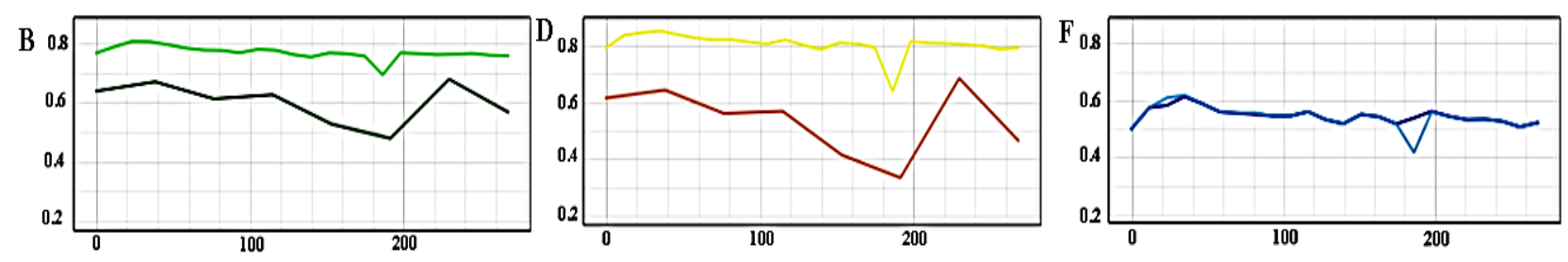

Fonte: Os autores (2021).

Apesar de o NDVI ser sensível às variações nas atividades fotossintéticas da vegetação, as quais estão diretamente relacionadas com a intensidade de absorção da radiação incidente na faixa espectral do vermelho, o GDVI por sua vez, utiliza a banda do verde e do infravermelho, isto é, duas bandas de baixa absorção na vegetação (MELO, 2019), justificando assim, os altos valores nos perfis em relação ao NDVI.

Os índices NDVI e GNDVI ainda apresentaram uma tendência a linearidade próxima para o perfil 1 (Figuras 5A e 5C) com exceção do perfil 2 (Figuras 5B e 5D). Essa tendência de estabilização para os valores dos índices também foi observada em outros estudos de comportamento vegetal (RAMBO et al., 2010; PONZONI; SHIMABUKURO, 2007), em que os autores descobriram que o aumento da biomassa leva à saturação nos valores do índice, em especial o NDVI, diminuindo a resposta dos índices com o avanço do desenvolvimento da vegetação.

O EVI por sua vez apresentou os menores valores para ambos os sensores quanto aos perfis e uma linearidade de valores e expressiva proximidade entre os sensores, (Figura 5F), com exceção ao perfil 1, mostrando apenas uma variação significativa (Figura 5E), em que assumiu altos valores, isto ocorreu, pois, a área analisada trata-se de uma região de confluência, o que costuma ser caracterizado pela presença de áreas de água e vegetação.

Ficou evidenciado que se tem um trecho com possível transição de ocupação, nas áreas de confluência do reservatório de Serrinha II para o Rio Pajeú à jusante. Neste perfil, os valores dos índices mostraram uma variabilidade, o EVI para o sensor MSI, assumiu valores altos, em alguns pontos superando o NDVI, que não manteve linearidade para a distribuição dos pixels ao longo do trecho em relação aos demais perfis e do GNDVI, que também apresentaram variações. O sensor OLI por sua vez tendeu a uma maior proximidade para os IVs baseados no Infravermelho. Isto pode ter ocorrido pela existência de mistura espectral entre os pixels e a área de confluência traçada no perfil. Em regiões semiáridas, um dos grandes problemas é a influência secundária do solo, bem como a mistura dos espectros solo-vegetação (CHAVES et al., 2013). Também é importante ressaltar que a resolução espacial é um elemento balizador a evidências de mistura espectral para a identificação de alvos. Independentemente das resoluções espaciais, os pixels de uma imagem de satélite tendem a apresentar uma mistura espectral, resultado de uma combinação da reflectância dos alvos naturais ou artificiais contidos neste (dois ou mais alvos) (FERREIRA; FERRIRA; FERREIRA, 2008). Resultados da influência de mistura espectral entre áreas de confluência em bacias do semiárido podem ser vistos com mais detalhes em Luiz, Martins e Couto (2019) e Francisco et al. (2012).

\subsubsection{VALIDAÇÃO DA CORRELAÇÃO ESTATÍSTICA ENTRE AS IMAGENS}

Com a finalidade de comparar as imagens OLI - Landsat 8 e MSI - Sentinel 2, a serem aplicadas no mapeamento das áreas de mata ciliar, foi gerada uma análise de correlação entre as respectivas imagens dos IVs: GNDVI e NDVI (Figura 6A e B), GNDVI e EVI (Figura 6C e D) e NDVI e EVI (Figura 6E e F), 
permitindo a comparação entre os produtos dos dois sensores através do coeficiente de determinação $\left(\mathrm{R}^{2}\right)$ e correlação de Pearson $(\alpha)$. Estas estatísticas foram utilizadas para avaliar o grau de correspondência linear entre os IVs (FREIRES et al., 2019).

O GNDVI e NDVI apresentaram boa correlação, com $\mathrm{R}^{2}=0,85$ para o sensor OLI e 0,88 para o MSI, e $\alpha=0,93$ para o OLI e 0,94 para o MSI. Ou seja, estatisticamente este resultado indica um comportamento de proporcionalidade direta entre os índices de vegetação nas feições matas ciliares.

Figura 6 - Correlação entre os IVs.
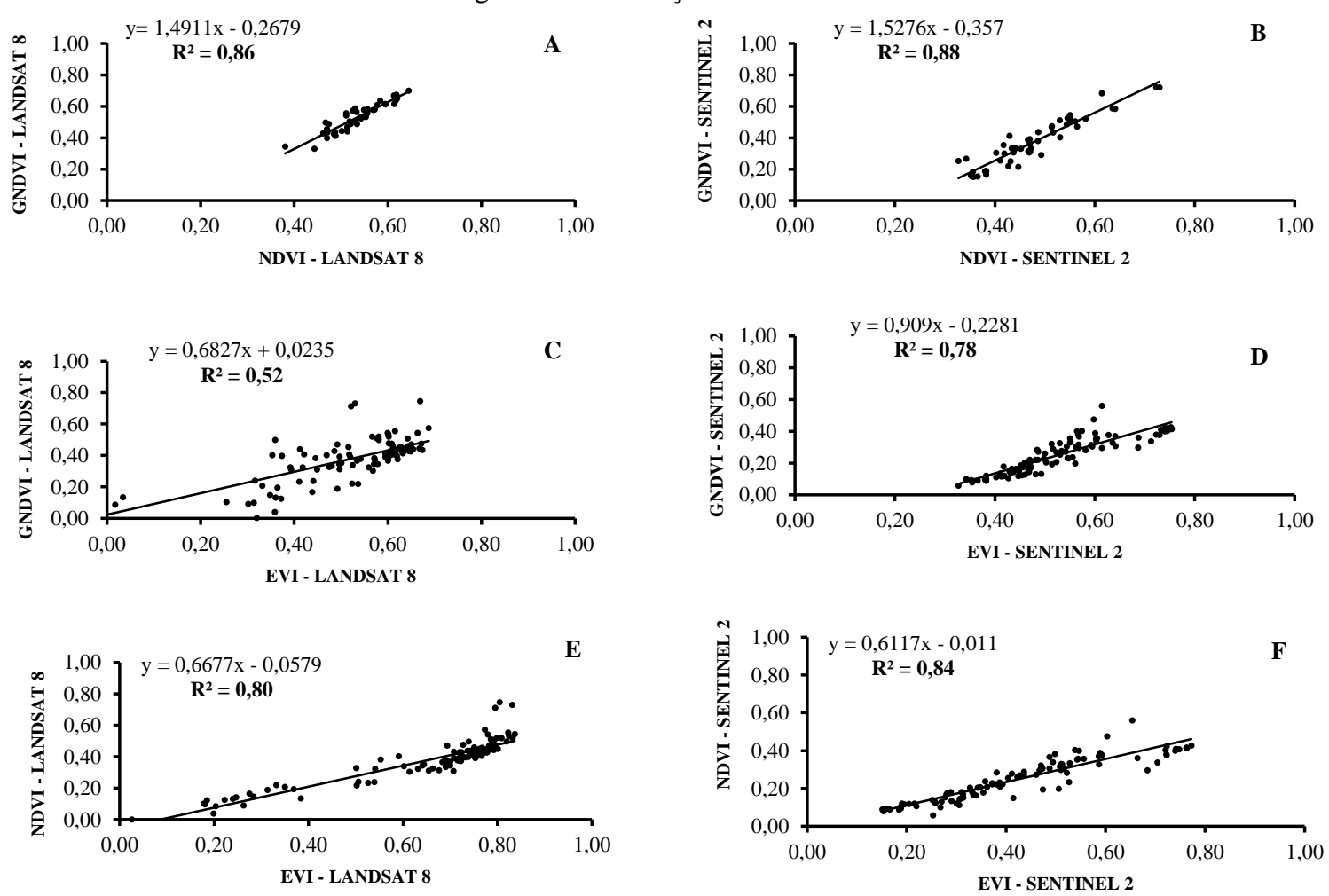

Fonte: Os autores (2021).

Os resultados indicaram que os índices GNDVI e EVI apresentaram baixo valor de $\mathrm{R}^{2}$ (Figura $6 \mathrm{C}$ e D) quando comparado aos demais, em especial para o sensor OLI, com valor de $\mathrm{R}^{2}=0,52$ e $\alpha=0,78$ e $\mathrm{R}^{2}=$ 0,78 e $\alpha=0,85$ para o sensor MSI, mostrando assim, baixa relação entre os IVs e os demais, em especial para o OLI. Esses resultados convergem aos encontrados por Tong e He (2017), Loranty et al. (2018), Fu-min et al. (2007) e Oliveira et al. (2017), que atribuiram as respostas às baixas concentrações de clorofila que são capazes de saturar a absorção na região azul.

Os resultados obtidos por Loranty et al. (2018) indicaram que o GNDVI e EVI não aumentaram consideravelmente com cobertura vegetal densa. Os únicos relacionamentos significativos que os autores encontraram houve correlações negativas entre a cobertura do dossel observada e os IVs no final da estação de crescimento da vegetação, e quando os IVs foram calculados em média durante a estação de crescimento usando a cobertura do dossel modelada em toda a paisagem produziu os mesmos resultados. Tong e He (2017) afirmaram a partir de seus resultados que, índices propostos para estimativa do estresse da vegetação usando comprimentos de onda no verde e /ou regiões azuis, demonstraram correlações baixas em relação aos demais IVs.

Este resultado se expande ao correlacionar os indices NDVI e EVI (Figura 6 E e F), com valor de R $^{2}$ $=0,80$ e $\alpha=0,89$ para o sensor OLI e $\mathrm{R}^{2}=0,84$ e $\alpha=0,93$ para o sensor MSI. Chaves et al. (2013), obtiveram coeficientes de correlações consideráveis para o EVI e NDVI. Tais resultados podem ter relação ao fato do EVI ter sido projetado para ter maior faixa dinâmica, assim resolver o déficit conhecido de NDVI sendo saturado em altos níveis de biomassa, por exemplo, mais de 0,8 (WARDLOW; EGBERT, 2010). 


\subsubsection{ANÁLISE DA PRECISÃO DO MAPEAMENTO DAS ÁREAS DE MATA CILIAR E RESERVATÓRIO}

Para determinação das classes, obtidas partir da binarização para os alvos mata ciliar e reservatório (água), optou-se por uma imagem orbital de melhor resolução espacial do que as imagens OLI - Landsat 8, aplicada ao mapeamento. Nesse intuito foi utilizada a imagem MSI - Sentinel 2 como referência do mapeamento, que apresentou resultados satisfatórios em análises similares, desenvolvidas por Mondal, McDermid e Qadir (2020), Liu et al. (2020), Gibson et al. (2020) e Freires et al. (2019). Como resultado foi gerado a matriz de confusão ou de erros, onde se pode atestar a qualidade dos mapeamentos realizados (GIBSON et al. 2020; FREIRES et al., 2019), mostrando a distribuição de porcentagem de pixels classificados correta e erroneamente, a partir dos coeficientes estatísticos: Índice Kappa, que trata-se de um método estatístico que permite analisar de forma comparativa os resultados dos mapas obtidos por meio do sensoriamento remoto, dentro de certo limite (LANDIS; KOCH, 1977), omissão que representa o número de amostras que Exatidão Global, que representa a estimativa de acurácia (CONGALTON; GREEN, 2008). Os erros de omissão (EO) referem-se à probabilidade de que um valor numa determinada classe tenha sido classificado corretamente e os erros de comissão (EC) a probabilidade de que um valor previsto para estar numa determinada classe é realmente da classe (JI; LIU; BUCHROITHNER, 2018).

A Tabela 4 apresenta os valores extraídos da matriz de confusão da classificação gerada a partir dos IVs investigados e do SAVI, Exatidão Global, Índice Kappa, Erros de Omissão (EO) e Erros de Comissão (EC).

Tabela 4 - Acurácia das áreas classificadas.

\begin{tabular}{c|c|c|c|c|c|c}
\hline \multirow{2}{*}{ IVs } & Classe & Área $\left(\mathbf{k m}^{\mathbf{2}}\right)$ & $\mathbf{E O}(\boldsymbol{\%})$ & $\mathbf{E C}(\boldsymbol{\%})$ & Acurácia Global (\%) & $\begin{array}{c}\text { Índice } \\
\boldsymbol{k a p p a}(\boldsymbol{k})\end{array}$ \\
\hline \multirow{2}{*}{ GNDVI } & MATA CILIAR & 1,67 & 55,55 & 8,75 & 96,25 & 0,58 \\
& ÁGUA & 7,37 & 0,92 & 1,02 & 98,33 & 0,92 \\
\hline \multirow{2}{*}{ NDVI } & MATA CILIAR & 2,38 & 45,65 & 16,51 & 96,23 & 0,64 \\
& ÁGUA & 6,80 & 1,20 & 0,90 & 98,15 & 0,91 \\
\hline \multirow{2}{*}{ EVI } & MATA CILIAR & 0,51 & 10,00 & 11,74 & 99,30 & 0,76 \\
& ÁGUA & 8,09 & 0,14 & 0,46 & 99,48 & 0,97 \\
\hline \multirow{2}{*}{ SAVI (Ls =0,25) } & MATA CILIAR & 0,03 & 76,44 & 10,97 & 98,91 & 0,40 \\
& ÁGUA & 7,38 & 3,25 & 9,61 & 98,77 & 0,93 \\
\hline \multirow{2}{*}{ SAVI (Ls =0,50) } & MATA CILIAR & 1,29 & 45,95 & 26,46 & 98,85 & 0,64 \\
& ÁGUA & 7,18 & 2,26 & 2,08 & 99,45 & 0,96 \\
\hline \multirow{2}{*}{ SAVI (Ls = 1,00) } & MATA CILIAR & 2,43 & 36,17 & 16,11 & 97,88 & 0,74 \\
& ÁGUA & 7,44 & 0,41 & 0,42 & 99,25 & 0,95 \\
\hline
\end{tabular}

Fonte: Os autores (2021).

Os resultados evidenciados na Tabela 4 indicaram que os IVs apresentaram resultados satisfatórios para os parâmetros analisados, variando de bom a excelentes para o Índice kappa. Esta conclusão baseia-se nas classes de desempenho do Índice kappa descritas por Landis e Koch (1977), em que valores menores ou iguais à zero têm-se um desempenho péssimo, $(0$ - 0,2) ruim, $(0,21$ - 0,4) razoável, $(0,41-0,6)$ bom, $(0,61$ - 0,8) muito bom e $(0,81-1,0)$ excelente. A acurácia global superou $95 \%$ para todos os IVs para as classes, o EVI aproximou-se da totalidade de porcentagem. Reflexo dos resultados obtidos para o índice atmosférico através das cartas imagem (Figura 3C), em que os resultados dos satélites OLI - Landsat 8 e MSI - Sentinel 2 apresentaram forte proximidade.

O Índice Kappa foi alto para maior parte das classificações, isto se deve ao fato de que os resultados dos classificadores condizem com a realidade. Para a mata ciliar o Índice Kappa variou o desempenho de bom a muito bom, sendo o menor valor para o GNDVI (bom) e maior para EVI (muito bom). A classe reservatório (água) para todos os índices apresentou desempenho excelente, sendo o EVI o maior valor.

Estes resultados corroboram com os resultados obtidos com a binarização (Figura 4), onde para o ano de 2019 em todos os IVs foram apresentadas baixa presença de pixels que apesar de não representarem o alvo água, foram classificados devido ao intervalo estabelecido pelos limiares, o que influenciou também no resultados das áreas, apresentando alta proximidade entre os produtos dos respectivos satélites, tais resultados 
corroboram com os encontrados em Gibson et al. (2020) e Freires et al. (2019).

Ao analisar os dados de omissão e comissão, percebe-se que os IVs apresentaram maiores valores de omissão para a classe mata ciliar, possivelmente causados pela ausência de homogeneidade espacial da mata ciliar, e pela diferença de resolução espacial entre as imagens OLI - Landsat 8 e a imagem de referência MSI - Sentinel 2, neste sentido o GNDVI apresentou um maior valor de omissão para a classe $(55,55 \%)$ seguido do NDVI (45,65 \%). Em relação ao EC o NDVI teve maior valor de comissão (16,51 \%), o que indica ter havido pixels subestimados entre as áreas de floresta mais homogêneas e em fase de crescimento, típicas do período seco na Caatinga. O EVI por sua vez apresentou os menores valores de omissão e comissão (10 e $11,74 \%)$ para a classe. Para a classe água, em ambos os erros (EC e EO), os IVs variaram entre 0 e 1,5\%, apontando que nenhum ou poucos pixels da classe mata ciliar, tenham sido inseridos nessa feição, ou ainda que quase todos os pixels da classe água foram rotulados corretamente.

Para a classe mata ciliar, o índice Kappa foi diretamente proporcional a variação do parâmetro de ajuste ao solo, variando de razoável a muito bom (Tabela 4). Com relação ao EC o maior valor foi para Ls = $0,50(26,46 \%)$ e o $\mathrm{EO} \mathrm{Ls}=0,25(76,44 \%)$. Esses valores evidenciaram as afirmações de Huete (1988) para o Ls, ou seja, à medida que foi empregado um parâmetro $\mathrm{Ls}=1,00$ próximo ao cenário da vegetação da Caatinga no período seco, houve uma maior heterogeneidade entre as classes, e um aumento da acurácia do mapeamento. Ao ser empregado Ls $=0,50$ houve decréscimo na acurácia do mapeamento e do valor das áreas extraídas, que se expande em Ls $=0,25$. Para a classe água ambos os parâmetros apresentaram resultados próximos, com kappa entre 0,93 e 0,96 (excelente) e baixos valores de omissão e comissão.

Segundo o mapeamento realizado pelo Instituto Nacional de Pesquisa Espacial (INPE) no reservatório entre os anos de 2012 a 2017, através de imagens do satélite OLI - Landsat 8, houve ação de efeitos da seca no reservatório, em que o reservatório tem uma redução de área do espelho d'água de $25\left(\mathrm{~km}^{2}\right)$ em 2012, a valores inferiores a $5\left(\mathrm{~km}^{2}\right)$ (INPE, 2020), uma elevada mudança, onde esse efeito, teve ação direta na mata ciliar proporcionando como consequência sua redução.

\section{CONCLUSÃO}

Neste estudo, os resultados forneceram a compreensão de padrões espaço-temporais de uso do solo e do comportamento das áreas de mata ciliar do bioma Caatinga, num intervalo de 34 anos (1985 a 2019). A partir da análise da sensibilidade espectral dos IVs: GNDVI, NDVI e EVI, através de imagens dos satélites TM - Landsat 5, OLI - Landsat 8 e MSI - Sentinel 2. Em que foram extraídas as seguintes conclusões:

Em termos espaciais, houve a identificação de possíveis eventos contínuos de seca, o que proporcionou a redução da área superficial do reservatório e da mata ciliar ao longo dos anos.

Os índices de vegetação do infra-vermelho (GNDVI e NDVI) apresentaram maior sensibilidade para classificar a mata ciliar. O EVI e o SAVI $\left(\mathrm{L}_{S}=1,0\right)$ apresentaram maior acurácia de mapeamento, evidenciando os resultados presentes na literatura, em que são indicados para biomas semiáridos. Entretanto, foi identificada a potencialidade dos demais IVs em realizar mapeamentos destas áreas com eficiência, possibilitando efetuar monitoramentos de ações características da vegetação da Caatinga. Porém, as respostas obtidas através dos IVs devem ser interpretadas com cautela, considerando os diversos fatores influenciadores nos alvos, como: ações atmosféricas, climáticas e topografia do terreno.

Contudo, destaca-se a utilidade dos dados da série Landsat e do satélite Sentinel 2 possibilitando análises espaciais e espectrais no âmbito das características de cada satélite, para entender o comportamento dos IVs na dinâmica da Caatinga através do mapeamento da mata ciliar no entorno de reservatórios.

\section{Agradecimentos}

A Universidade Federal de Pernambuco (UFPE) por meio do Departamento de Engenharia Cartográfica (Decart), Departamento de Engenharia Civil e Ambiental (Deciv) e do Programa de PósGraduação em Ciências Geodésicas e Tecnologias da Geoinformação (PPGCGTG), que proporcionaram o desenvolvimento desta pesquisa. Ao Projeto de Pesquisa intitulado "Coberturas vegetal e hídrica de bacias hidrográficas utilizando imagens orbitais no estado de Pernambuco", aprovado na Chamada Universal 
MCTIC/CNPq 2018 - FAIXA A, processo $n^{\circ} 433914 / 2018-1$, da terceira autora. O segundo autor agradece ao projeto $\mathrm{PQ/nível} \mathrm{2/CNPq} \mathrm{n}^{\circ}$ do Proc.310452/2018-0. Por fim, a Fundação de Amparo à Ciência e Tecnologia do Estado de Pernambuco (FACEPE) pelo incentivo a pesquisa e concessão de bolsa de iniciação científica do quarto autor.

\section{Contribuição dos Autores}

O primeiro autor participou nas etapas de conceptualização, desenvolvimento metodológico, processamento, ajustamento dos dados e redação textual. O segundo autor foi responsável pela conceptualização, processamento e análises dos dados, revisão e correção textual. O terceiro autor foi responsável pela análise dos dados, adequações metodológicas e revisão textual. E o quarto autor atuou nas etapas de aquisição de dados, processamento e ajustamento dos dados.

\section{Conflitos de Interesse}

Os autores declaram que não há conflitos de interesse.

\section{Referências}

AGÊNCIA PERnAMbUCANA DE ÁGUAS E CLIMA (APAC). Boletim de Monitoramento Pluvio. Recife, 2020. Disponível em: < http://www.apac.pe.gov.br/meteorologia/monitoramento-pluvio.php>. Acesso em: 5 mai. 2020.

agÊnCia nacional de ÁguAs e SANEAMEnTo (ANA). Portal HidroWeb - Séries Históricas. Recife, 2020. Disponível em: < http://www.snirh.gov.br/hidroweb >. Acesso em: 10 mai. 2020.

ABADE, N.A.; JÚNIOR, O.A.C.; GUIMARÃES, R.F.; DE OLIVEIRA, S.N. Comparative Analysis of MODIS Time-Series Classification Using Support Vector Machines and Methods Based upon Distance and Similarity Measures in the Brazilian Cerrado-Caatinga Boundary. Remote Sensing, [s.1.], v.7, n.1, p.12160-12191, Sep 2015. DOI. 10.3390/rs70912160

ALI, A.; MARTELLI, R.; LUPIA, F.; BARBANTI, L. Assessing Multiple Years' Spatial Variability of Crop Yields Using Satellite Vegetation Indices. Remote Sensing, [s.1.], v. 11, n. 20, p. 2384-2406, 15 out. 2019. DOI. 10.3390/rs11202384.

AREKHI, M.; GOKSEL, C.; SANLI, F. B.; SENEL, G. Comparative Evaluation of the Spectral and Spatial Consistency of Sentinel-2 and Landsat-8 OLI Data for Igneada Longos Forest. ISPRS International Journal Of Geo-information, [s.1.], v. 8, n. 2, p. 56-73, 28 Jan 2019. DOI. 10.3390/ijgi8020056.

BATISTA, J. .; FRANCEZ, B.M.L.; ELISIANE ALBA, E.; SCHUH, S.M.; FAVARIN, S.A.J.; SILVA, O.J.; HOFIÇO, S.N.; PEREIRA, S. R.;SEBEM, E. Comportamento Espectral de Paricá (Schizolobium parahyba var. amazonicum (Huber ex Ducke Barneby) em Plantios com Diferentes Idades. Anuário do Instituto de Geociências - UFRJ. Rio de Janeiro, Vol. 41, n.3, p. 82-95, Sep 2018. DOI. 10.11137/2018_3_82_95.

BARBOSA, H. A.; KUMAR, T. V. L.; PAREDES, F.; ELLIOTT, S.; AYUGA, J.G. Assessment of Caatinga response to drought using Meteosat-SEVIRI Normalized Difference Vegetation Index (2008-2016). ISPRS Journal of Photogrammetry and Remote Sensing, [s.1.], v. 148, p. 235-252, Feb 2019. Elsevier BV. DOI. 10.1016/j.isprsjprs.2018.12.014.

BECK, P.S.A., ATZBERGER, C., HOGDA, K.A., JOHANSEN, B., SKIDMORE, A.K. Improved monitoring of vegetation dynamics at very high latitudes: a new method using MODIS NDVI. Remote Sensing Environ, [s.1.], V. 100, n.3, p.321-334, Feb 2006. DOI. 10.1016/j.rse.2005.10.021.

BISPO P. C.; VALERIANO, M. M.; DUPLICH, T. M. Relação da vegetação de caatinga com a condição geomorfométrica local. Revista Brasileira de Engenharia Agrícola e Ambiental, Campina Grande, 
v.14, n.5, p.523-530, May 2010. DOI. 10.1590/S1415-43662010000500010

BRASIL. Lei ${ }^{\circ}$ 12.727, de 17 de outubro de 2012. Institui o Novo Código Florestal Brasileiro. Diário Oficial da União, Brasília, DF, n. 227, 18 Oct 2012. Seção 1, p. 1.

CANDIAGO, S.; REMONDINO, F.; DE GIGLIO, MICHAELA; DUBBINI, M.; GATTELLI, M. Evaluating Multispectral Images and Vegetation Indices for Precision Farming Applications from UAV Images. Remote Sensing, [s.1.], v. 7, n.4, p. 4026-4047, Apr 2015. DOI. 10.3390/rs70404026

CHAVES, I. DE B.; LOPES, V. L.; FFOLLIOTT, P. F.; PAES-SILVA, A. P. Uma classificação morfoestrutural para descrição e avaliação da biomassa da vegetação da caatinga. Revista Caatinga, Mossoró, v.21, n.2, p.204-213, Jun 2008. ISSN. 0100-316X.

CHAVES, I. DE B.; FRANCISCO, P. R. M.; LIMA, E. R. V. DE; SILVA, B. B. DA; BRANDAO, Z. N.; CHAVES, L. H. G. Índices espectrais, diagnóstico da vegetação e da degradação da Caatinga da Bacia do Rio Taperoá-PB. Embrapa Algodão. Relatório Técnico. 2013. Disponível em < https://www.embrapa.br/busca-de-publicacoes/-/publicacao/977328/indices-espectrais-diagnostico-davegetacao-e-da-degradacao-da-caatinga-da-bacia-do-rio-taperoa-pb >. Acesso em: 06 março 2020.

CHAVEZ JR., P.S. An improved dark-object subtraction technique for atmospheric scattering correction of multispectral data. Remote Sensing of Environment, [s.1.], v.24, n.3, p. 459- 479, Apr 1988. DOI. 10.1016/0034-4257(88)90019-3.

CLAVERIE, M., MASEK, J.G., JU, J., DUNGAN, J.L., Harmonized Landsat-8 Sentinel-2 (HLS) Product User's Guide. National Aeronautics and Space Administration (NASA), Washington. Disponível em: < https://hls.gsfc.nasa.gov/wp-content/uploads/2017/08/HLS.v1.3.UserGuide_v2.pdf>. Acesso em: 11 Jul 2020.

CRESPO-MENDES, N.; LAURENT, A.; HENRIKBRUUNB, H.; HAUSCHILDA, Z. M. Relationships between plant species richness and soil $\mathrm{pH}$ at the level of biome and ecoregion in Brazil. Ecological Indicators, [s.1.], v. 98, n.0, p. 266-275, Mar 2019. DOI. 10.1016/j.ecolind.2018.11.004.

COELHO, R. C. T. P.; BUFFON, I.; GUERRA, T. Influência do uso e ocupação do solo na qualidade da água: um método para avaliar a importância da zona ripária. Ambi-Agua, Taubaté, v. 6, n. 1, p. 104-117, 2011. DOI. 10.4136/1980-993X.

CONGALTON, R.G.; GREEN, K. Assessing the Accuracy of Remotely Sensed Data: principles and practices. 2 ed. New York, CRC Press. 2008.

DRUSCH, M.; BELLO, U. del; CARLIER, S.; COLIN, O.; FERNANDEZ, V.; GASCON, F.; HOERSCH, B.; ISOLA, C.; LABERINTI, P.; MARTIMORT, P. Sentinel-2: ESA's optical high-resolution mission for GMES operational services. : ESA's Optical High-Resolution Mission for GMES Operational Services. Remote Sensing Of Environment, [s.1.], v. 120, p. 25-36, May 2012. DOI. 10.1016/j.rse.2011.11.026.

EUROPEAN SPATIAL AGENCY (ESA). Copernicus: Sentinel-2 - The Optical Imaging Mission for Land Services. 2020. Disponível em: <https://directory.eoportal.org/web/eoportal/satellite-missions/cmissions/copernicus-sentinel-2\#sensors>. Acesso em: 22 mai. 2020.

FERREIRA, G. L.; FERREIRA, C. N.; FERREIRA, E. M. Sensoriamento remoto da vegetação: evolução e estado da arte. Acta Scientiarum. Biological Sciences, Goiás, v. 30, n.4 p. 379-390, Nov 2008. DOI. 10.4025/actascibiolsci.v30i4.5868.

FRANCISCO, P. R. M. Modelo de mapeamento da deterioração do Bioma Caatinga da bacia hidrográfica do Rio Taperoá, PB. 97f. Tese (Doutorado em Engenharia Agrícola) - Centro de Tecnologia e Recursos Naturais, Universidade Federal de Campina Grande, 2013.

FU-MIN, W.; JING-FENG, H.; YAN-LIN, T.; XIU-ZHEN, W. New Vegetation Index and Its Application in Estimating Leaf Area Index of Rice. Rice Science, [s.1.], v.14, n.3, Sep 2007. DOI. 10.1016/S16726308(07)60027-4.

FU, P., WENG, Q. Consistent land surface temperature data generation from irregularly spaced Landsat imagery. Remote Sensing Environment, [s.1.], v.184, p.175-187. Oct 2016. DOI. 
10.1016/j.rse.2016.06.019.

FREIRES, E. V.; SILVA, C. Â.; CUNHA, D. S. R.; DUARTE, C. R.; VERÍSSIMO, C. U. V.; GOMES, D. D. M.. Comparison of OLI/ Landsat 8 and MSI/Sentinel-2 Images in Cover and Land Use Mapping in the Uruburetama Massif, Ceará. Anuário do Instituto de Geociências - UFRJ, [s.1.], v. 42, n. 4, p. 427-442, 12 Dec 2019. DOI. 10.11137/2019_4_427_442.

GHOLIZADEH, A.; ŽIŽALAA, D.; SABERIOON, M.; BORŏVKA, L. Soil organic carbon and texture retrieving and mapping using proximal, airborne and Sentinel-2 spectral imaging. Remote Sensing of Environment, [s.1.], v. 218, p. 89-103, Dec 2018. DOI. 10.1016/j.rse.2018.09.015.

GIBSON, R.; DANAHER, T.; HEHIR, W.; COLLINS, L. A remote sensing approach to mapping fire severity in south-eastern Australia using sentinel 2 and random forest. Remote Sensing Of Environment, [s.l.], v. 240, p. 111702-111714, Apr 2020. DOI. 10.1016/j.rse.2020.111702.

GITELSON, A. A.; KAUFMAN, Y. J.; MERZLYAK, M. N. Use of a green channel in remote sensing of global vegetation from EOS-MODIS. Remote Sensing of Environment, [s.1.], v.58, n.3, p.289-298, Dec 1996. DOI. 10.1016/S0034-4257(96)00072-7.

GUIMARÃES, A. P. Dinâmica da resposta espectral da vegetação de caatinga na bacia hidrográfica do açude Soledade, utilizando técnicas de sensoriamento remoto. $106 \mathrm{f}$. Dissertação (Mestrado em Geografia) - Universidade Federal da Paraíba. Areia, 2009.

HABOUDANE, D.; MILLER, J.R.; PATERRY, E.; ZARCO-TEJADA, P.J.; STRACHAN, I.B. Hyperspectral vegetation indices and novel algorithms for predicting green LAI of crop canopies: modeling and validation in the context of precision agriculture. Remote Sensing of Environment, [s.l.], v. 90, n. 3, p. 337-352, Apr 2004. DOI. 10.1016/j.rse.2003.12.013.

HELMAN, D.; BAHAT, I.; NETZER, Y.; BEN-GAL, A.; ALCHANATIS, V.; PEETERS, A.; COHEN, Y. Using Time Series of High-Resolution Planet Satellite Images to Monitor Grapevine Stem Water Potential in Commercial Vineyards. Remote Sensing, [s.1.], v.10, n.10, p.1010-1615, Oct 2018. DOI. 10.3390/rs10101615.

HÉNAUlT-ETHIER, L.; DUSSAUlT, M.; CABRERA, P.; LEFEBVRE, B.; TAILlEFER, S.; DESCHAMPS, M.; VANDENBERG, G. Les insectes au service de l'humain pour la gestion des résidus organiques. Paris. Vecteur Environnement. Relatório técnico 2017. Disponível em: < https://www.researchgate.net/publication/318883142_Entotechnologie_Les_insectes_au_service_de_l'hu main_pour_la_gestion_des_residus_organiques> Acesso em: 26 março 2020.

HUETE, A. R. A soil ajusted vegetation index (SAVI). Remote Sensing Environment, [s.1.], v.25, n.3, p.295309, Aug 1988. DOI. 10.1016/0034-4257(88)90106-X.

HUETE, A.R.; DIDAN, K.; MIURA, T.; RODRIGUEZ, E.P.; GAO, X.; FERREIRA, L.G. Overview of the radiometric and biophysical performance of the MODIS vegetation indices. Remote Sensing Environment, [s.1.], v. 83, n.1-2, p.195-213, Nov 2002. DOI. 10.1016/S0034-4257(02)00096-2.

HUETE, A. R.; KAMEL, D.; SHIMABUKURO, Y. E.; RATANA, P.; SALESKA, S. R.; HUTYRA, L. R.; YANG, W.; NEMANI, R. R.; MYNENI, R. Amazon rainforests green-up with sunlight in dry season. Geophysical Research Letters, [s.1.], v. 33, N. 6405, p.1-4, Mar 2006. DOI. 10.1029/2005GL025583.

INSTITUTO NACIONAL DE PESQUISAS ESPACIAIS (INPE). Recursos Hídricos e Reservatórios Mapa do Reservatório de Serrinha II. São José dos Campos, 2020. Disponível em: <http://geopro.crn.inpe.br/RH_reser_PE_serrinhaII.htm >. Acesso em: 10 May. 2020.

JARCHOW, J.C.; DIDAN, K.; BARRETO-MUNOZZ, A.; NAGLER, L. P.; GLENN, P.E.; Application and Comparison of the MODIS-Derived Enhanced Vegetation Index to VIIRS, Landsat 5 TM and Landsat 8 OLI Platforms: A Case Study in the Arid Colorado River Delta, Mexico. Sensors, [s.1.], v.18, n.5, p.1546, May 2018. DOI. 10.3390/s18051546.

JENSEN, J.R. Sensoriamento Remoto do Ambiente - Uma Perspectiva em Recursos Terrestres. São José dos Campos: Parêntese Editora, 2009. 598p.

JI, M.; LIU, L.; BUCHROITHNER, M. Identifying Collapsed Buildings Using Post-Earthquake Satellite 
Imagery and Convolutional Neural Networks: A Case Study of the 2010 Haiti Earthquake. Remote Sensing, [s.1.], v.10, 11, p.1-20, Oct 2018. DOI. 10.3390/rs10111689.

KIMM, H.; GUAN, K.; JIANG, C.; PENG, B.; GENTRY, L. F.; WILKIN, SCOTT C.; WANG, S.; CAI, Y.; BERNACCHI, C. J.; PENG, JIAN. Deriving high-spatiotemporal-resolution leaf area index for agroecosystems in the U.S. Corn Belt using Planet Labs CubeSat and STAIR fusion data. Remote Sensing of Environment, [s.1.], v. 239, p. 111615-111627, Mar 2020. DOI. 10.1016/j.rse.2019.111615.

LANDIS, J. R.; KOCH, G. G. The Measurement of Observer Agreement for Categorical Data. Biometrics, [s.1.], v. 33, n. 1, p. 159-174, Mar 1977. DOI. 10.2307/2529310.

LIU, W.; HUANG, J.; WEI, C.; WANG, X.; MANSARAY, L. R.; HAN, J.; ZHANG, D.; CHEN, Y. Mapping water-logging damage on winter wheat at parcel level using high spatial resolution satellite data. ISPRS Journal of Photogrammetry and Remote Sensing, [s.1.], v. 142, p. 243-256, Aug 2018. Elsevier BV. DOI. 10.1016/j.isprsjprs.2018.05.024.

LIU, L.; XIAO, X.; QIN, Y.; WANG, J.; XU, X.; HU, Y.; QIAO, Z. Mapping cropping intensity in China using time series Landsat and Sentinel-2 images and Google Earth Engine. Remote Sensing Of Environment, [s.1.], v. 239, p. 111624-111636, Mar 2020. DOI. 10.1016/j.rse.2019.111624.

LUIZ, P. G.; MARTINS, R. P.; COUTO JUNIOR, F. A. variação interanual da cobertura da terra de uma ottobacia no médio curso do Rio Araguaia. In: Simpósio de Brasileiro de Sensoriamento Remoto. 19, 2019, Santos. Anais... São Paulo: Instituto Nacional de Pesquisas Espaciais, 2019, p. 1426-1429.

LORANTY, M. M.; DAVYDOV, P.S.; HEATHER KROPP, H.; ALEXANDER, D. H.; MACK, C. M.; NATALI, M.S. ; NIKITA, Z. S. Vegetation Indices Do Not Capture Forest Cover Variation in Upland Siberian Larch Forests. Remote Sensing, [s.1.], V.10, n.0, p.1686, Oct 2018. DOI. 10.3390/rs10111686.

MACINTYRE, P.; NIEKERK, V. A.; MUCINA, L; Efficacy of multi-season Sentinel-2 imagery for compositional vegetation classification. International Journal of Applied Earth Observation and Geoinformation, Gangtok, V. 85, n.1, p. 0303-2434, Marc 2020. DOI. 10.1016/j.jag.2019.101980.

MARIANO, D. A.; DOS SANTOS, C. A. C.; WARDLOW, B. D.; ANDERSON, M. C.; SCHILTMEYER, A. V.; TADESSE, T.; SVOBODA, M. D.. Use of remote sensing indicators to assess effects of drought and human-induced land degradation on ecosystem health in Northeastern Brazil. Remote Sensing of Environment, [s.1.], v. 213, p. 129-143, Aug 2018. DOI. 10.1016/j.rse.2018.04.048.

MELAAS, E.K., FRIEDL, M.A., ZHU, Z. Detecting interannual variation in deciduous broadleaf forest, phenology using Landsat TM/ETM+ data. Remote Sensing Environment, [s.1.], v.132, p.176-185. May 2013. DOI. 10.1016/j.rse.2013.01.011.

MELO, B. D. M.; MIRANDA, S. R.P.; GONÇALVES, S.T.; DE ASSIS, L. A.; CHRISTIAN DIAS CABACINHA, D.C.; MARCATTI, E. G.; CASTRO, O.V.R.; ARAUJO JUNIOR, A.C. Análise temporal do NDVI como ferramenta para o planejamento do ecoturismo na APA do Rio Pandeiros, norte de Minas Gerais. Caderno de Ciências agrárias, Minas Gerais, v.11, n.0, p.01-08, Sep 2019. ISSN. 2447-6218.

MERCIER, A.; BETBEDER, J.; BAUDRY, J.; LE ROUX, V.; SPICHER, F.; LACOUX, J.; ROGER, D.; HUBERT-MOY, L. Evaluation of Sentinel-1 \& 2 time series for predicting wheat and rapeseed phenological stages. ISPRS Journal of Photogrammetry and Remote Sensing, [s.1.], v. 163, p. 231256, Mar 2020. DOI. 10.1016/j.isprsjprs.2020.03.009.

MERRICK, T.; PAU, S.; JORGE, P. S. L. M.; SILVA, F.S.T.; BENNARTZ, R. Spatiotemporal Patterns and Phenology of Tropical Vegetation Solar-Induced Chlorophyll Fluorescence across Brazilian Biomes Using Satellite Observations. Remote Sensing, [s.1.], v.11, n.1746, p.1-26, Jul 2019. DOI. 10.3390/rs11151746.

MEYER, Lorenz Hans; HEURICH, Marco; BEUDERT, Burkhard; PREMIER, Joseph; PFLUGMACHER, Dirk. Comparison of Landsat- 8 and Sentinel-2 Data for Estimation of Leaf Area Index in Temperate Forests. Remote Sensing, [s.1.], v. 11, n. 10, p. 1160-1176, May 2019. DOI. 10.3390/rs11101160.

MONDAL, P.; MCDERMID, S. S.; QADIR, A. A reporting framework for Sustainable Development Goal 15: multi-scale monitoring of forest degradation using modis, landsat and sentinel data. : Multi-scale monitoring of forest degradation using MODIS, Landsat and Sentinel data. Remote Sensing Of 
Environment, [s.1.], v. 237, p. 111592-111606, Feb 2020. DOI. 10.1016/j.rse.2019.111592.

MORAIS, Y. C. B.; SANTOS, B. O.; LAURENTINO, M. L. S.; SILVA, J. C. B.; GALVÍNCIO, J. D. Análise espaço-temporal e detecção de mudanças da cobertura vegetal no município de Floresta, PE - Brasil, utilizando o NDVI. In: Simpósio de Brasileiro de Sensoriamento Remoto. 15, 2011, Curitiba. Anais... São Paulo: Instituto Nacional de Pesquisas Espaciais, 2019, p. 2128-2134.

MYNENI, R.B; HOFFMAN, S; KNYAZIKHIN, Y; PRIVETTE, J.L; GLASSY, J; TIAN, Y; WANG, Y; SONG, X; ZHANG, Y; SMITH, G.R. Global products of vegetation leaf area and fraction absorbed PAR from year one of MODIS data. Remote Sensing Of Environment, [s.1.], v. 83, n. 1-2, p. 214-231, Nov 2002. DOI. 10.1016/s0034-4257(02)00074-3.

NAVARRO, G.; ISABEL CABALLERO, I.; SILVA, G.; PEDRO-CECILIO PARRA, P.; VÁZQUEZD, A.; CALDEIRA, R. Evaluation of forest fire on Madeira Island using Sentinel-2A MSlimagery. International Journal of Applied Earth Observation and Geoinformation, [s.1.], v. 58, p. 97-106, Jun 2017. DOI. 10.1016/j.jag.2017.02.003.

OLIVEIRA, R. F. L.; OLIVEIRA, R. L. M.; GOMES, S. F.; SANTANA, C. R. Estimating foliar nitrogen in Eucalyptus using vegetation indexes. Scientia Agricola, Piaracicaba, v.74, n.2, p.142-147, Apr 2017. DOI. 10.1590/1678-992x-2015-0477.

PASTOR-GUZMAN, J.; DASH, J.; ATKINSON, P. M. Remote sensing of mangrove forest phenology and its environmental drivers. Remote Sensing Of Environment, [s.1.], v. 205, p. 71-84, Feb 2018. Elsevier BV. DOI. 10.1016/j.rse.2017.11.009.

PAREDES-TREJO, F.; BARBOSA, A. H.; SPATAFORA, R. L. Assessment of SM2RAIN-Derived and Stateof-the-Art Satellite Rainfall Products over Northeastern Brazil. Remote Sensing, [s.1.], v.10, n.1093, p. 1 - 29, Jul 2018. DOI. 10.3390/rs10071093

PEREIRA, M. P. S.; JUSTINO, F.; MALHADO, A. C. M.; BARBOSA, H.; MARENGO, J. The influence of oceanic basins on drought and ecosystem dynamics in Northeast Brazil. Environmental Research Letters, [s.1.], v. 9, n. 12, p. 124013-124020, Dec 2014. DOI. 10.1088/1748-9326/9/12/124013.

PONZONI, F. J.; SHIMABUKURO, Y. E. Sensoriamento remoto no estudo da vegetação. São José dos Campos: INPE, 2007. 127 p.

QI, J.; CHEHBOUNI, A.; HUETE, A.R.; KERR, Y.H.; SOROOSHIAN, S.. A modified soil adjusted vegetation index. Remote Sensing Of Environment, [s.1.], v. 48, n. 2, p. 119-126, May 1994. DOI. 10.1016/0034-4257(94)90134-1.

RAMBO, L.; MA, B. L.; XIONH, Y.; SILVIA, P. R. F. Leaf and canopy optical characteristics as crop-Nstatus indicators for field nitrogen management in corn. Journal of Plant Nutrition and Soil Science, Temuco, v. 173, n.3, p. 434-443, Jun 2010. DOI. 10.1002/jpln.200900022.

ROUSE, J.W.; HASS, R.H., JR.; SCHELL, J.A.; DEERING, D.W. Monitoring vegetation systems in the great plains with ERTS. In: Proceedings of the Third Earth Resources Technology Satellite-1 Symposium 1 (A), Texas A\&M Univ, College Station. Anais... TX, USA, 1974; pp. 309-317.

SKAKUN, S., KUSSUL, N., SHELESTOV, A., KUSSUL, O., Flood hazard and flood risk assessment using a time series of satellite images: a case study in Namibia. Risk Anal., [s.l.], v. 3, n.8, p.1521-1537. Aug 2014. DOI. 10.1111/risa.12156.

SHAO, Z.; CAI, J.; FU, P.; HU, L.; LIU, T. Deep learning-based fusion of Landsat-8 and Sentinel-2 images for a harmonized surface reflectance product. Remote Sensing of Environment, [s.1.], v. 235, p.111425111442, Sept 2019. DOI. 10.1016/j.rse.2019.111425.

SILVA, M. T.; AZEVEDO, P. V.; SILVA, V. P. R.; SILVA, B. K. N.; MARIANO, E. B.; AMORIM, M. R. B. Estimativa da produção de sedimentos na bacia hidrográfica do submédio Rio São Francisco. Journal of Environmental Analysis and Progress, Recife, v. 2, p. 203 - 2011, Jun 2017. DOI. 10.24221/jeap.2.3.2017.1430.203-211.

TESTA, S.; SOUDANI, K.; BOSCHETTI, L.; MONDINO, B. E. MODIS-derived EVI, NDVI and WDRVI time series to estimate phenological metrics in French deciduous forests. International Journal Of 
Applied Earth Observation And Geoinformation, [s.l.], v. 64, n.1 p.132-144, Aug 2018. DOI. 10.1016/j.jag.2017.08.006.

TONG, A.; HE, Y. Estimating and mapping chlorophyll content for a heterogeneous grassland: comparing prediction power of a suite of vegetation indices across scales between years. ISPRS Journal Of Photogrammetry And Remote Sensing, [s.1.], v. 126, p. 146-167, Apr 2017. DOI. 10.1016/j.isprsjprs.2017.02.010. 16/j.jag.2017.08.006

UNITED STATES GEOLOGICAL SURVEY (USGS). The Earth Explore. Disponível em: < http://earthexplorer.usgs.gov/>. Acesso em: 5 mai. 2020.

VALERA, C.A.; PISSARRA, T.C.T.; FILHO, M.V.M.; VALLE JÚNIOR, R.F.; OLIVEIRA, C.F.; MOURA, J.P.; SANCHES FERNANDES, L.F.; PACHECO, F.A.L. The Buffer Capacity of Riparian Vegetation to Control Water Quality in Anthropogenic Catchments from a Legally Protected Area: A Critical View over the Brazilian New Forest Code. Water, [s.1.], v. 11, n. 3, p.549-565, Mar 2019. DOI. 10.3390/w11030549.

WARDLOW, B.D.; EGBERT, S.L. A comparison of MODIS 250-m EVI and NDVI data for crop mapping: A case study for southwest Kansas. International Journal of Remote Sensing, [s.1.], v.31, n.3, p.805830, Feb 2010. DOI. 10.1080/01431160902897858.

WHITE, J.C., WULDER, M.A., HERMOSILLA, T., COOPS, N.C., HOBART, G.W. A nationwide annual characterization of 25 years of forest disturbance and recovery for Canada using Landsat time series. Remote Sensing Environment, [s.1.], v.194, p.303-321. Jun 2017. DOI. 10.1016/j.rse.2017.03.035.

YANG, S.; BAI, J.; ZHAO, C.; LOU, H.; ZHANG, C.; GUAN, Y.; ZHANG, Y.; WANG, Z.; YU, X. The assessment of the changes of biomass and riparian buffer width in the terminal reservoir under the impact of the South-to-North water diversion project in China. Ecol. Indic., Coimbra, v. 85, n.1, p.932-943, Feb 2018. DOI. 10.1016/j.ecolind.2017.11.011.

ZHANG, J.; LI, S.; DONG, R.; JIANG, C.; NI, M. Influences of land use metrics at multi-spatial scales on seasonal water quality: A case study of river systems in the Three Gorges Reservoir Area, China. Journal of Cleaner Production, [s.1.], v.206, n.1, p.76-85, Jan 2019. DOI. 10.1016/j.jclepro.2018.09.179.

ZHOU, F.; ZHONG, D. Kalman filter method for generating time-series synthetic Landsat images and their uncertainty from Landsat and MODIS observations. Remote Sensing of Environment, [s.1.], v. 239, p. 111628-111653, Mar 2020. DOI. 10.1016/j.rse.2019.111628.

\section{Biografia do autor principal}

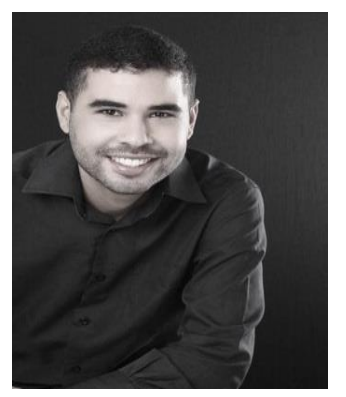

Ubiratan Joaquim da Silva Junior, natural de Recife-PE. Engenheiro Cartógrafo formado pela Universidade Federal de Pernambuco (UFPE) e Mestrando em Ciências Geodésicas e Tecnologias da Geoinformação (PPGCGTG) pela mesma instituição. Atualmente, atua como pesquisador em Sensoriamento Remoto e Geodésia Ambiental, no citado Programa de Pós-Graduação. Tem experiência em Cartografia e Geodésia, atuando principalmente nos seguintes temas: monitoramento costeiro, Hidrologia, Sensoriamento Remoto e Recursos Hídricos, com ênfase em Tecnologia Ambiental.

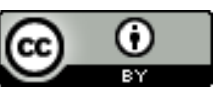

Esta obra está licenciada com uma Licença Creative Commons Atribuição 4.0 Internacional - CC BY. Esta licença permite que outros distribuam, remixem, adaptem e criem a partir do seu trabalho, mesmo para fins comerciais, desde que lhe atribuam o devido crédito pela criação original. 